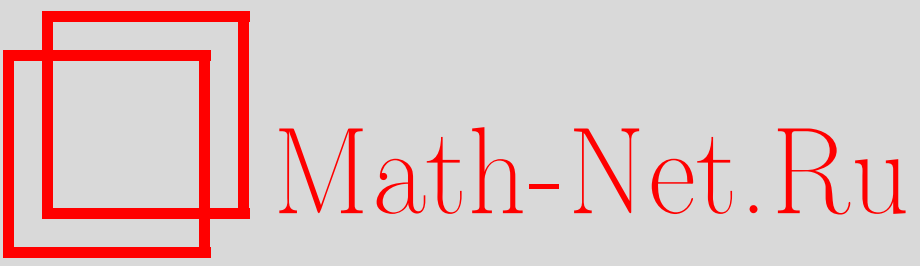

А. А. Борисенко, Внешняя геометрия сильно параболических многомерных подмногообразий, УМH, 1997, том 52, выпуск 6, 3-52

DOI: https://doi.org/10.4213/rm891

Использование Общероссийского математического портала Math-Net.Ru подразумевает, что вы прочитали и согласны с пользовательским соглашением

http://www.mathnet.ru/rus/agreement

Параметры загрузки:

IP: 3.93 .64 .190

26 апреля 2023 г., 15:15:05 


\title{
ВНЕШНЯЯ ГЕОМЕТРИЯ СИЛЬНО ПАРАБОЛИЧЕСКИХ МНОГОМЕРНЫХ ПОДМНОГООБРАЗИЙ
}

\author{
А. А. БОРИСЕнко \\ СОДЕРЖАНИЕ
}

1. Введение

2. Аффинная классификация точек многомерных поверхностей $\ldots \ldots \ldots \ldots .4$

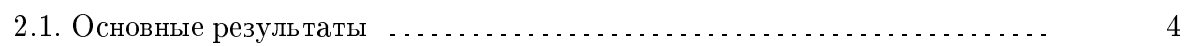

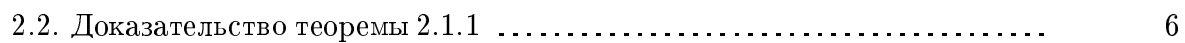

2.3. Классификация точек трехмерных подмногообразий .............. 11

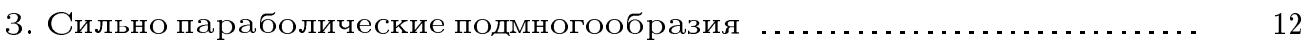

3.1. Локальное строение сильно параболической поверхности в римановом пространстве .........................................

3.2. Локалњное строение сильно параболической поверхности в евклидовом пространстве ......................................

3.3. Полные сильно параболические многомерные поверхности в евклидовом

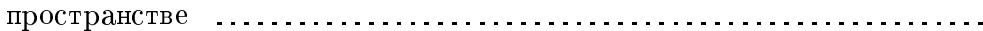

3.4. Вполне геодезичность полных сильно параболических подмногообразий в сферическом пространстве

3.5. Сильно параболические подмногообразия в пространстве Лобачевского

3.6. Сильно параболические подмногообразия в псевдоевклидовом и псевдоримановом пространствах постоянной кривизны . . . . . . . . . . . . . . .

3.7. Сильно параболические подмногообразия и минимальные поверхности

\section{1. Введение}

Хорошо известно, что в случае двумерных поверхностей в трехмерном евклидовом пространстве классы поверхностей тесно связаны с классами римановых метрик. Выпуклые, седловые, параболические регулярные поверхности несут индуцированную метрику соответственно неотрицательной, неположительной, нулевой гауссовой кривизны. Справедливы, в некотором смысле, обратные теоремы о локальном изометрическом погружении двумерных римановых метрик положительной гауссовой кривизны, отрищательной гауссовой кривизны, нулевой гауссовой кривизны в классе регулярных выпуклых, седловых, параболических поверхностей в трехмерном евклидо- 
вом пространстве. Имеются и глобальные теоремы в этом направлении, доказанные А. Д. Александровым, А. В. Погореловым, И. В. Ефимовьм, Э. Г. Позняком.

Однако в многомерном случае таких тесных связей нет. Известна теорема Неша и Кейпера о локальном изометрическом погружении произвольного риманового многообразия в виде гладкой класса $C^{1}$ гиперповерхности в евклидово пространство. Однако при этом теряются всякие связи между внутренней и внешней геометрией. И в многомерном случае геометрия разделилась на две ветви: риманова геометрия и геометрия многомерных подмногообразий в различных объемлюших пространствах.

В настояшем обзоре дается описание некоторых классов многомерных подмногообразий произвольной коразмерности в различных объемлюших пространствах, аналогичных классу параболических поверхностей в $\mathbb{E}^{3}$. Изучается их локальное и глобальное метрическое строение, топологическое строение.

Этот обзор написан на основе лекций, которые прочитаны автором в 1995-1996 гг. в университете города Бразилиа (Бразилия). Автор выражает сердечную благодарность профессору Кети Тененблат за приглашение и гостеприимство.

\section{2. Аффинная классификация точек многомерных поверхностей}

2.1. Основные результаты. Хорошо известна классификация точек регулярной двумерной поверхности в трехмерном евклидовом пространстве. Выделяются три невырожденных класса точек: гиперболические - в них гауссова кривизна отрицательна, эллиптические - гауссова кривизна положительна, параболические - с нулевой гауссовой кривизной, и один вырожденньй класс - точки уплощения, в них обе главные кривизны равны нулю. Таким же образом можно классифицировать точки на регулярных гиперповерхностях в евклидовом пространстве: класс точки определяется знаками главных кривизн поверхности.

В связи с этим естественно поставить вопрос о классификации точек регулярной поверхности $F^{l}$, вложенной в евклидово пространство $\mathbb{E}^{n}$ с произвольной коразмерностью.

Вопрос о разбиении точек поверхности $F^{l}$ на классы, устойчивые относительно некоторой группы преобразований пространства, был поставлен С. З. Шефиелем в работе [28].

Согласно результатам работы [30], существует четыре типа геометрических групп преобразований, т.е. групп, содержаших в качестве собственной подгрупшы групу подобия евклидова пространства. Это група аффинных преобразований, группа конформных преобразований, группа преобразований, сохраняюших отношение объемов, и общая группа - довольно обширная группа диффеоморфизмов пространства $\mathbb{E}^{n}$. Из работы [30] следует, что только группы аффинных и конформных преобразований сохраняют локально внешнюю геометрию, а стало быть, только эти группы могут служить основой классификации точек поверхности $F^{l} \subset \mathbb{E}^{n}$.

Мы будем изучать классификацию точек относительно группы аффинных преобразований пространства $\mathbb{E}^{n}$.

ОПРЕДЕЛЕНИЕ. Две точки регулярной поверхности, вложенной в евклидово пространство, назьваются аффинно әквивалентными , если соприкасающиеся параболоиды в этих точках можно отобразить друг на друга невырожденным афффинньм преобразованием в объемлющем евклидовом пространстве. 
В работах [28], [29] указаны некоторые аффинные типы точек двумерных поверхностей в евклидовом пространстве произвольной размерности. Полная аффинная классификация точек поверхности $F^{2}$ в $\mathbb{E}^{4}$ и ее связь с классификацией по кривизне грассманова образа поверхности $F^{2}$ приведена в [16].

Мы будем назьвать точечной коразмерностью поверхности $F^{l} \subset \mathbb{E}^{n}$ в данной точке размерность пространства вторых квадратичных форм поверхности $F^{l}$ в этой точке. Очевидно, что точечная коразмерность $p$ не превьшает $l(l+1) / 2$ и, вообще говоря, не равна абсолютной коразмерности $n-l$.

Имеет место

ТеОРема 2.1.1. Пусть $F^{l} \subset \mathbb{E}^{n}$ - регулярная l-мерная поверхность, вложсенная в $n$-мерное евклидово пространство $(l \geqslant 2)$. Тогда точки поверхности можнно разбить на конечное число классов аффинной эквивалентности только в следующих случаях:

1) для двумерньх поверхностей произвольной точечной коразмерности,

2) для гиперповерхностей $F^{l} \subset \mathbb{E}^{l+1}$ и двойственного случая $F^{l} \subset \mathbb{E}^{n}$, где точечная коразмерность $p=l(l+1) / 2-1$,

3) для поверхностей $F^{l} \subset \mathbb{E}^{n}$, где точечная коразмерность минимальна $p=0$ или максимальна $p=l(l+1) / 2$,

4) для трехмерных поверхностей $F^{l} \subset \mathbb{E}^{n}$, где точечная коразмерность равна 2 или 4.

В случае 1) сушествуют восемь аффинных классов:

1) $p=0$, один класс - точка уплощения,

2) $p=1$, три класса - эллиптический, гиперболический, параболический,

3) $p=2$, три класса; соприкасающийся параболоид невырожденным афффинным преобразованием объемлющего пространства приводится к одному из следующих канонических видов:

а) $x^{3}=2 x^{1} x^{2}, x^{4}=\left(x^{2}\right)^{2}$,

б) $x^{3}=\left(x^{1}\right)^{2}, x^{4}=\left(x^{2}\right)^{2}$,

в) $x^{3}=2 x^{1} x^{2}, x^{4}=\left(x^{1}\right)^{2}-\left(x^{2}\right)^{2}$,

4) $p=3$, один класс - свободная точка.

В случае 2) при $p=1$ и $p=l(l+1) / 2-1$ для четного $l=2 m$ выделяются $(m+1)^{2}-1$ различных аффинных классов, для нечетного $l=2 m-1$ выделяются $m(m+1)-1$ аффиинных классов.

В случае 3$)$ имеется один класс: при $p=0$ - точка уплошения, при $p=l(l+1) / 2$ свободная точка.

В этом случае любые две точки подмногообразия аффинно эквивалентны, так как в этом случае в каждой точке пространство вторых квадратичных форм изоморфно линейному пространству симметричных квадратных матриц порядка $l$.

В случае 4$)$ как при $p=2$, так и при $p=4$ существует десять классов, не сводяшихся к аффинным классам точек двумерных поверхностей.

По нашему мнению, тот факт, что в ситуации общего положения имеется бесконечное число аффинных классов, является причиной того, что в многомерном случае нет такой тесной связи, как в двумерном, между классами римановых метрик и классами поверхностей в евклидовом пространстве, в которых естественно изометрическое 
погружение этих метрик. Кроме того, эта теорема объясняет, почему в изучении подмногообразий наибольшее продвижение достигнуто в случае двумерных поверхностей и гиперповерхностей.

Для поверхности $F^{l}$ в евклидовом пространстве $\mathbb{E}^{n}$ определяется внешний нуль-индекс $\mu(Q)$ в точке $Q \in F^{l}$. Он равен размерности максимального подпространства $L(Q)$ в касательном пространстве $T_{Q} F^{l}$, такого, что для любого $y \in L(Q)$ вьполняется условие $A y=0$, где $A$ - матрица второй квадратичной формы поверхности $F^{l}$ относительно произвольной нормали в точке $Q$.

ОПРЕДЕЛЕНИЕ. Направление $y$ в касательном пространстве $T_{Q} F^{l}$ назьвается асимптотическим относительно нормали $N$, если $\left(A_{N} y, y\right)=0$, где $A_{N}-$ матрица второй квадратичной формы поверхности $F^{l}$ в точке $Q$ относительно нормали $N$; направление $y \in T_{Q} F^{l}$ назьвается асимптотическим направлением поверхности $F^{l}$ в точке $Q$, если оно асимптотическое относительно любой нормали.

Классификация точек поверхности $F^{3} \subset \mathbb{E}^{5}$ дается следующей теоремой.

ТеОРема 2.1.2. Две точки регулярной поверхности с нулевьм внешним нуль-индексом являются аффинно әквивалентньми тогда и только тогда, когда

1) пучки вторьх квадратичных форм поверхности в этих точках имеют равные числа и степени линейных и квадратичных әлементарных делителей,

2) количества асимптотических направлений поверхности в этих точках совпадают.

Выделяется десять невырожденных аффинных классов точек на поверхности $F^{3} \subset \mathbb{E}^{5}$ (см. табл. 1).

Если нуль-индекс отличен от нуля, то к этим классам добавляются:

а) 3 класса с точечной коразмерностью 2 , которые локально с точностью до членов второго порядка соответствуют вложению $F^{3}=F^{2} \times \mathbb{E}^{1} \subset \mathbb{E}^{5}$ или $F^{2} \subset \mathbb{E}^{4}$,

б) 3 класса с точечной коразмерностью 1 , которые порождаются аффинньми классами двумерных поверхностей в $\mathbb{E}^{3}$,

в) 1 класс с точечной коразмерностью, равной нулю, отвечаюший точке уплощения.

2.2. Доказательство теоремы 2.1.1. Пусть $Q$ и $Q^{\prime}$ - точки на регулярной поверхности $F^{l} \subset \mathbb{E}^{l+p}$. Если в пространстве $\mathbb{E}^{l+p}$ выбрать систему координат так, чтобы точка $Q$ совпадала с началом координат $O$, а касательное пространство $T_{Q} F^{l}$ - с подпространством $\left\{x^{1}, \ldots, x^{l}\right\}$, то уравнение соприкасающегося параболоида к поверхности $F^{l}$ в точке $Q$ будет следуюшим:

$$
\left\{x^{l+\mu}=\left\langle A^{\mu} x, x\right\rangle\right\}_{\mu=1}^{p},
$$

где $x=\left(x^{1}, \ldots, x^{l}\right),\langle\cdot, \cdot\rangle$ - скалярное произведение в $\mathbb{E}^{l}$, а $A^{\mu}(x, x)(\mu=1, \ldots, p)$ компоненты вектора второй квадратичной формы $H$ поверхности $F^{l}$ в точке $Q$,

$$
H=\left(A^{1}(x, x), A^{2}(x, x), \ldots, A^{p}(x, x)\right),
$$


ТАБЛИЦА 1

\begin{tabular}{|c|c|c|c|}
\hline № & $\begin{array}{c}\text { Элементарные } \\
\text { делители }\end{array}$ & $\begin{array}{c}\text { Число } \\
\text { асимптотич. } \\
\text { направлений }\end{array}$ & $\begin{array}{c}\text { Канонический вид } \\
\text { пучка вторых } \\
\text { квадратичных форм }\end{array}$ \\
\hline $1 \mathrm{a}$ & $\lambda-\lambda_{1}, \lambda-\lambda_{2}$ & 0 & $\left(x^{2}\right)^{2}+\left(x^{3}\right)^{2},\left(x^{1}\right)^{2}+\left(x^{3}\right)^{2}$ \\
\hline $1 б$ & $\lambda-\lambda_{3}, \lambda_{1} \neq \lambda_{2} \neq \lambda_{3}$ & 4 & $-\left(x^{2}\right)^{2}+\left(x^{3}\right)^{2},-\left(x^{1}\right)^{2}+\left(x^{3}\right)^{2}$ \\
\hline $2 \mathrm{a}$ & $\lambda-\lambda_{1}, \lambda-\lambda_{1}$ & 0 & $\left(x^{3}\right)^{2},\left(x^{1}\right)^{2}+\left(x^{2}\right)^{2}$ \\
\hline $2 б$ & $\lambda-\lambda_{3}, \lambda_{1} \neq \lambda_{3}$ & 2 & $\left(x^{3}\right)^{2},-\left(x^{1}\right)^{2}+\left(x^{2}\right)^{2}$ \\
\hline $3 \mathrm{a}$ & $\lambda-\lambda_{1}, \lambda-\lambda_{1}$ & 0 & $0,\left(x^{1}\right)^{2}+\left(x^{2}\right)^{2}+\left(x^{3}\right)^{2}$ \\
\hline $3 б$ & $\lambda-\lambda_{1}$ & $\infty$ & $0,\left(x^{1}\right)^{2}+\left(x^{2}\right)^{2}-\left(x^{3}\right)^{2}$ \\
\hline 4 & $\begin{array}{c}\left(\lambda_{1}, \lambda^{2}-2 \alpha \lambda+\right. \\
2\end{array} \beta^{2} ; \beta \neq 0$ & 2 & $-\left(x^{2}\right)^{2}+2 x^{2} x^{3}+\left(x^{3}\right)^{2}$, \\
\hline $5 \mathrm{a}$ & $\left(\lambda-\lambda_{1}\right)^{2}$ & 1 & $\left(x^{1}\right)^{2}+\left(x^{2}\right)^{2}+2 x^{2} x^{3}-\left(x^{3}\right)^{2}$ \\
\hline $5 б$ & $\lambda-\lambda_{3} ; \lambda_{1} \neq \lambda_{3}$ & 3 & $\left(x^{2}\right)^{2}-\left(x^{3}\right)^{2}, 2 x^{1} x^{3}-\left(x^{2}\right)^{2}$ \\
\hline 6 & $\left(\lambda-\lambda_{1}\right)^{2}, \lambda-\lambda_{1}$ & 1 & $\left(x^{2}\right)^{2}, 2 x^{1} x^{2}+\left(x^{3}\right)^{2}$ \\
\hline 7 & $\left(\lambda-\lambda_{1}\right)^{3}$ & 2 & $2 x^{2} x^{3},\left(x^{2}\right)^{2}+2 x^{1} x^{3}$ \\
\hline 8 & пунус вырожден & 1 и & $2 x^{2} x^{3}, 2 x^{1} x^{3}$ \\
\hline 1 параб. $)$ & плоскость & \\
\hline
\end{tabular}

$A^{\mu}$ - симметрическая вешественная $(l \times l)$-матрица. Везде в дальнейшем будем отождествлять квадратичную форму с соответствующей ей симметрической матрицей. Таким образом, соприкасаюшийся параболоид полностью описьвается второй квадратичной формой поверхности $F^{l}$ в точке $Q$. Если $H^{\prime}=\left\{A^{\prime 1}, A^{\prime 2}, \ldots, A^{\prime p}\right\}$ - векторная вторая квадратичная форма поверхности $F^{l}$ в точке $Q^{\prime}$, то после параллельного переноса на вектор $Q^{\prime} Q$ и поворота в $\mathbb{E}^{l+p}$ так, чтобы $T_{Q} F^{l}$ и $T_{Q^{\prime}} F^{l}$ совпали, уравнение соприкасающегося параболоида в $Q^{\prime}$ будет иметь вид

$$
\left\{x^{l+\mu}=\left\langle A^{\prime \mu} x, x\right\rangle\right\}_{\mu=1}^{p}
$$

Точки $Q$ и $Q^{\prime}$ аффинно эквивалентны, если существует $\Lambda \in G L(l+p, \mathbb{R})$ такое, что $\Lambda H=H^{\prime}$. Можно показать, что для наших целей достаточно ограничиться рассмотрением преобразований $\Lambda$, которые имеют блочньй вид

$$
\Lambda=\left(\begin{array}{cc}
U^{-1} & 0 \\
0 & V
\end{array}\right)
$$

где $U \in G L(l, \mathbb{R})$ и $V \in G L(p, \mathbb{R})$ - афффинные преобразования в касательном $T_{O} F^{l}$ и в нормальном $N_{O} F^{l}$ пространствах в точке О к поверхности $F^{l}$, т.е. $\Lambda \in G L(l, \mathbb{R}) \times$ $G L(p, \mathbb{R})$. 
Если $H=\left(A^{1}, \ldots, A^{p}\right)$, то

$$
\Lambda H=V\left(\begin{array}{c}
U^{*} A^{1} U \\
\vdots \\
U^{*} A^{p} U
\end{array}\right), \text { т.е. }(\Lambda H)^{\nu}=U^{*}\left(v_{\mu}^{\nu} A^{\mu}\right) U
$$

где $v_{\mu}^{\nu}$-элементы матрицы $V, U^{*}$ - матрища, транспонированная к $U$.

Действительно, в выбранной системе координат по теореме о неявной функции поверхность задается уравнениями

$$
\left\{x^{l+\mu}=f^{\mu}\left(x^{1}, \ldots, x^{l}\right)\right\}_{\mu=1}^{p},
$$

причем функции $f^{\mu}$ регулярны,

$$
f^{\mu}(O)=0,\left.\quad \frac{\partial f^{\mu}}{\partial x^{i}}\right|_{0}=0 \quad(\mu=1, \ldots, p ; i=1, \ldots, l)
$$

Тогда компоненты второй квадратичной формы поверхности $F^{l}$ в точке О равны $A_{i j}^{\mu}=\left.\frac{\partial^{2} f^{\mu}}{\partial x^{i} \partial x^{j}}\right|_{0}$. Если аффинное преобразование $\Lambda$ в $\mathbb{E}^{l+p}$ сохраняет на месте касательное пространство

$$
T_{O} F^{l}=\left(x^{1}, x^{2}, \ldots, x^{l}\right)
$$

то его матрица имеет вид

$$
\left(\begin{array}{cc}
U^{-1} & 0 \\
W & V
\end{array}\right)
$$

где $U \in G L(l, \mathbb{R}), V \in G L(p, \mathbb{R}), W-(p \times l)$-матрища. Пусть $x=\Lambda y$-рассматриваемое аффинное преобразование. Тогда уравнение поверхности $F^{l}$ запишется так:

$$
\left\{\begin{array}{c}
\left(\begin{array}{c}
y^{1} \\
\vdots \\
y^{l}
\end{array}\right)=U^{-1}\left(\begin{array}{c}
x^{1} \\
\vdots \\
x^{l}
\end{array}\right), \\
y^{l+1}=w_{i}^{1} x^{i}+v_{\mu}^{1} f^{\mu}\left(x^{1}, \ldots, x^{l}\right), \\
\ldots \ldots \ldots \ldots \ldots \ldots \ldots \ldots \ldots \ldots \ldots \ldots \\
y^{l+p}=w_{i}^{p} x^{i}+v_{\mu}^{p} f^{\mu}\left(x^{1}, \ldots, x^{l}\right),
\end{array}\right.
$$

где $w_{i}^{\nu}$ и $v_{\mu}^{\nu}(\mu, \nu=1, \ldots, p ; i=1, \ldots, l)$ - элементы матриц $W$ и $V$.

Компоненты второй квадратичной формы поверхности $F^{l}$ в начале координат после преобразования $\Lambda$ суть

$$
A_{i j}^{\prime \mu}=\frac{\partial^{2} y^{l+\mu}}{\partial y^{i} \partial y^{j}}=u_{j}^{k} u_{i}^{m} \frac{\partial^{2} y^{l+\mu}}{\partial x^{k} \partial x^{m}}=u_{j}^{k} u_{i}^{m} \frac{\partial^{2}\left(v_{\nu}^{\mu} f^{\nu}\right)}{\partial x^{k} \partial x^{m}}=u_{j}^{k} u_{i}^{m} v_{\nu}^{\mu} A_{k m}^{\nu}
$$

где $u_{j}^{k}(k, j=1, \ldots, l)$ - элементы матрищы $U$. 
Таким образом, афффинными классами точек поверхности являются классы эквивалентности пространства $\mathscr{H}=\{H\}$ всевозможных векторных вторых квадратичных форм (т.е. $p$-мерных векторов симметрических $(l \times l)$-матриц) по действию групш

$$
G=G L(l, \mathbb{R}) \times G L(p, \mathbb{R}),
$$

определенному формулой (2.2.1). Значит, $\mathscr{H} / G=\mathscr{H} / G L(l, \mathbb{R}) \times G L(p, \mathbb{R})-$ пространство аффинных классов. Пространство $\mathscr{H} / G L(p, \mathbb{R})$ - это пространство всевозможных $p$-мерных плоскостей $l(l+1) / 2$-мерного пространства $L$ симметрических $(l \times l)$-матрищ, т.е.

$$
\mathscr{H} / G L(p, \mathbb{R})=G(p, l(l+1) / 2),
$$

где $G(m, n)$ - многообразие Грассмана $m$-мерных плоскостей в $n$-мерном евклидовом пространстве. Поэтому

$$
\operatorname{dim} \mathscr{H} / G L(p, \mathbb{R})=p(l(l+1) / 2-p) .
$$

Дальнейший ход доказательства идейно аналогичен рассуждениям Р. Тома, применяемьм в теории особенностей $[4$, с. 55$]$. Для того чтобы число аффинных классов было конечно, необходимо, чтобы $\operatorname{dim} \mathscr{H} / G=0$. Отсюда

$$
\operatorname{dim} \mathscr{H} / G L(p, \mathbb{R}) \leqslant \operatorname{dim} G L(l, \mathbb{R}) .
$$

Это неравенство можно усилить, замечая, что подгруппа $\{\lambda \mathbf{I} \mid \lambda \in \mathbb{R} \backslash\{0\}\} \subset$ $G L(l, \mathbb{R})(\mathbf{I}$ - единичная матрица порядка $l)$ оставляет на месте $p$-мерную плоскость в пространстве $L$ симметрических $(l \times l)$-матрищ, натянутую на $A^{1}, A^{2}, \ldots, A^{p}$ (это следует из формулы (2.2.1)). Поэтому множество аффинных классов совпадает с фактор-пространством

$$
\mathscr{H} /\left(G L(p, \mathbb{R}) \times G L(l, \mathbb{R}) /\{\lambda \mathbf{I}\}_{\lambda \neq 0}\right) .
$$

Нетрудно видеть, что $G L(l, \mathbb{R}) /\{\lambda \mathbf{I}\}=S L(l, \mathbb{R})$ и $\operatorname{dim} G L(l, \mathbb{R}) /\{\lambda \mathbf{I}\}=l^{2}-1$.

Итак, чтобы число аффинных классов было конечно, необходимо, чтобы

$$
\operatorname{dim} \mathscr{H} / G L(p, \mathbb{R}) \leqslant \operatorname{dim} S L(l, \mathbb{R}),
$$

значит,

$$
p(l(l+1) / 2-p) \leqslant l^{2}-1 .
$$

Это неравенство имеет следуюшие решения:

I. $l=2$;

II. $p=1$ или $p=l(l+1) / 2-1, l \geqslant 2$;

III. $p=0$ или $p=l(l+1) / 2, l \geqslant 2$;

IV. $l=3, p=2$ или $p=4$. 
Рассмотрим их.

I. Для двумерной поверхности $F^{2} \subset \mathbb{E}^{n}$ в зависимости от точечной коразмерности $p$ имеем:

1. $p=0$. Пространство $\mathscr{H}$ нульмерно - все точки аффинно эквивалентны. Точки этого аффинного класса - точки уплощения.

2. $p=1$. Пространство $\mathscr{H}$ одномерно. Имеем поверхность $F^{2} \subset \mathbb{E}^{3}$. В зависимости от знака гауссовой кривизны выделяем три класса: эллиптический $(K>0)$, параболический $(K=0)$ и гиперболический $(K<0)$.

3. $p=2$. Имеем поверхность $F^{2} \subset \mathbb{E}^{4}$. Пусть $\lambda_{1}, \lambda_{2}-$ корни уравнения $\operatorname{det}\left(A^{1}-\lambda A^{2}\right)=0$. Возможны три случая:

а) корни вешественны и различны;

б) корни вешественны и совпадают;

в) корни комплексно сопряжены.

Тогда элементарные делители пучка $A^{1}-\lambda A^{2}$ примут соответственно следующий вид:

a) $\lambda-\lambda_{1}, \lambda-\lambda_{2}, \lambda_{1} \neq \lambda_{2} ; \lambda_{1}, \lambda_{2} \in \mathbb{R}$

б) $\left(\lambda-\lambda_{1}\right)^{2} ; \lambda_{1} \in \mathbb{R}$

в) $\lambda_{2}-2 \alpha \lambda+\left(\alpha^{2}+\beta^{2}\right)$, где $\alpha, \beta \in \mathbb{R}, \beta \neq 0$.

Оказьвается, что элементарные делители полностью определяют аффинньй класс точки двумерной поверхности, для которой $p=2$. Ход рассуждений аналогичен ходу доказательства теоремы 2 из [4]. Канонический вид соприкасающегося параболоида для каждого афффинного класса приведен в формулировке теоремы 1 из [4].

4. $p=3$. Пространство $\mathscr{H}$ имеет максимальную размерность 3 . Поэтому уже группа $G L(3, \mathbb{R})$ действует на $\mathscr{H}$ транзитивно. Выделяется один аффинный класс - свободные точки. Канонический вид соприкасаюшегося параболоида таков: $z^{1}=\left(x^{1}\right)^{2}$, $z^{2}=\left(x^{2}\right)^{2}, z^{3}=x^{1} x^{2}$.

II. В случае $p=1$ имеем $F^{l} \subset \mathbb{E}^{l+1}$ - гиперповерхность. Пространство $\mathscr{H}$ одномерно: $\mathscr{H}=L$. Группа $G L(p, \mathbb{R})$ является попросту мультипликативной группой $\mathbb{R} \backslash\{0\}$. Поэтому пространство аффинных классов есть $L / G L(l, \mathbb{R})$. Пусть $A$ - вторая квадратичная форма поверхности $F^{l}$ в рассматриваемой точке $q ; k, s$ и $m$ - число ее положительных, отрицательных и нулевых собственных чисел (т.е. главных кривизн поверхности $F^{l}$ в точке $q$ ) соответственно и $j=\min \{k, s\}$. Тогда числа $j$ и $m$ дают полную систему аффинных инвариантов. При этом $0 \leqslant m \leqslant l-1,1 \leqslant j \leqslant(l-m+1) / 2$. Комбинаторньм подсчетом убеждаемся, что число аффинных классов соответствует значениям, приведенным в теореме 2.1.1.

Пусть теперь $p=l(l+1) / 2-1$. Тогда $H=\left(A^{1}, \ldots, A^{p}\right)$. Введем в пространстве $L$ симметрических $(l \times l)$-матриц скалярное произведение

$$
(B, C)=\operatorname{Tr}(B C),
$$

где $\operatorname{Tr}$ - след матрицы, и рассмотрим матрицу $A \in L$, ортогональную гиперплоскости, натянутой на $\left(A^{1}, \ldots, A^{p}\right)$. Поставим матрицу $A$ в соответствие векторной второй квадратичной форме $H$.

Под действием групшы $G L(p, \mathbb{R})$ гиперплоскость $\left(A^{1}, \ldots, A^{p}\right) \subset L$ остается на месте, поэтому $A$ не меняется. Под действием преобразования $U$ из групшы $G L(l, \mathbb{R})$ гиперплоскость $\left(A^{1}, \ldots, A^{p}\right)$ переходит в гиперплоскость $\left(U^{*} A^{1} U, \ldots, U^{*} A^{p} U\right) \subset L, \mathrm{a}$ 
ортогональная к ней матрица есть матрица $U^{-1} A U^{-1 *}$. Действительно,

$$
\operatorname{Tr}\left(U^{*} A^{\mu} U U^{-1} A U^{-1 *}\right)=\operatorname{Tr}\left(U^{*} A^{\mu} A U^{-1 *}\right)=\operatorname{Tr}\left(A U^{-1 *} U^{*} A^{\mu}\right)=\operatorname{Tr}\left(A A^{\mu}\right)=0 .
$$

Поэтому аффинная классификация точек поверхности $F^{l} \subset \mathbb{E}^{n}$ при $p=l(l+1) / 2-1$ сводится к классификашии при $p=1$.

III. Пусть $p=l(l+1) / 2$. Уже $\mathscr{H} / G L(p, \mathbb{R})$ состоит из одного элемента. Все вторые квадратичные формы (и все точки поверхности $F^{l}$ ) аффинно эквивалентны между собой. Соприкасающийся параболоид приводится к такому каноническому виду:

$$
\begin{aligned}
x^{l+1}=\left(x^{1}\right)^{2}, x^{l+2}=2 x^{1} x^{2}, x^{l+3} & =2 x^{1} x^{3}, \ldots, \\
x^{2 l} & =2 x^{1} x^{l}, x^{2 l+1}=\left(x^{2}\right)^{2}, \ldots, x^{l+p}=\left(x^{l}\right)^{2} .
\end{aligned}
$$

При $p=0$ пространство векторных форм $\mathscr{H}$ нульмерно и все точки аффинно эквивалентны.

$\mathrm{IV} . \mathrm{B}$ случае $l=3, p=2$ или $p=4$ доказательство дается теоремой 2.1.2.

Доказательство теоремы 2.1.2 можно найти в [14].

2.3. Классификация точек трехмерных подмногообразий. Для трехмерных подмногообразий $F^{3}$ в евклидовом пространстве $\mathbb{E}^{5}$ сушествует классификация точек подмногообразия по грассманову образу. Она почти эквивалентна аффинной классификации с той лиш разнищей, что в некоторых случаях одному грассманову типу соответствует два аффинных типа. Это аналогично тому, что для двумерных поверхностей в $\mathbb{E}^{3}$ эллиптические и гиперболические точки не отличимы по их сферическому образу, если не учитьвать степень сфферического отображения. Поэтому, чтобы осуществить полную классификацию точек трехмерных поверхностей по грассманову образу, необходимо ввести дополнительное понятие индекса.

Многообразием Грассмана $G(l, l+p)(l, p \geqslant 1)$ назьвается множество $l$-мерных плоскостей в евклидовом пространстве $\mathbb{E}^{l+p}$, проходяших через начало координат $O \in \mathbb{E}^{l+p}$.

Пусть $F^{l} \subset \mathbb{E}^{l+p}$ - регулярная класса $C^{2} l$-мерная поверхность в евклидовом пространстве. Построим в каждой точке поверхности касательное пространство и перенесем все эти пространства параллельно в начало координат. Полученное подмножество в $G(l, l+p)$ назьвается грассмановым образом поверхности $F^{l}$, отображение

$$
\Gamma: F^{l} \rightarrow G(l, l+p)
$$

описанное вьше, - грассмановым отображением.

Если в точке поверхности внешний нуль-индекс равен нулю (т.е. не существует ненулевого касательного вектора $X$ такого, что для любого касательного вектора $Y \alpha(X, Y)=0$, где $\alpha(X, Y)$ - векторная вторая квадратичная форма), то отображение $\Gamma$ в этой точке имеет максимальньй ранг. Мы будем предполагать это требование выполненным для всех точек поверхности $F^{l}$. Тогда грассманов образ будет невырожден: он будет регулярным $l$-мерным подмногообразием в $G(l, l+p)$.

Для двумерных поверхностей $F^{2} \subset \mathbb{E}^{4}$ Ю. А. Аминов вводил классификацию точек по кривизне $k$ многообразия Грассмана $G(2,4)$ по плошадке, касательной к грассманову образу поверхности; при этом выделялись точки, для которых 
a) $k>1$; б) $k=1$; в) $k<1[2]$.

Автором показана эквивалентность аффинной и грассмановой классификации.

Для трехмерных поверхностей $F^{3} \subset \mathbb{E}^{5}$ нас будет интересовать кривизна многообразия Грассмана $G(3,5), Q \in F^{3}$ по двумерным площадкам, касательным к $\Gamma\left(F^{3}\right)$ в точке $\Gamma(Q) \in G(3,5)$. При $l=3$ касательное пространство $T_{\Gamma(Q)} \Gamma\left(F^{3}\right)$ трехмерно, поэтому сужение тензора кривизны многообразия Грассмана $G(3,5)$ на это пространство можно полностью охарактеризовать сужением на него же тензора Риччи многообразия $G(3,5)$. Дальше под тензором Риччи многообразия $G(3,5)$ будем понимать его сужение на касательное пространство к $\Gamma F^{3}$.

Пусть $k_{i}(i=1,2,3)$ есть главные кривизны Риччи многообразия $G(3,5)$ в точке $\Gamma(Q)$, где $Q$ - точка на регулярной поверхности $F^{3} \subset \mathbb{E}^{5}$. Они, как известно, содержат максимальную и минимальную кривизну $G(3,5)$ по плошадкам из $T_{\Gamma(Q)} \Gamma\left(F^{3}\right)$. Поскольку секционная кривизна $k$ многообразия Грассмана заключена в пределах $[0,2]$, то и главные кривизны будут лежать в этом отрезке. Имеет место

Tеорема 2.3.1 [16]. Пусть $F^{3} \subset \mathbb{E}^{5}$ - регулярная поверхность, $Q \in F^{3}$ и внеиний нуль-индекс поверхности $F^{3}$ в точке $Q$ равен нулю. Тогда в зависимости от типов әлементарных делителей пучка вторых квадратичных форм поверхности $F^{3}$ в точке $Q$ главные кривизны Риччи многообразия Грассмана $G(3,5)$ в точке $\Gamma(Q)$ по площадкам, касательным к грассманову образу $F^{3}$, удовлетворяют оценкам, приведенным в табл. 2.

Границы изменения главных кривизн при различных элементарных делителях различны, а случаи 2 и 8 отличаются тем, что двумерные площадки, на которых достигается кривизна единица, касаются различных вполне геодезических подмногообразий: в случае 2 - трехмерной сферы $S^{3}=G(3,4) \subset G(3,5)$, в случае 8 - двумерной сферы $S^{2}=G(1,3) \subset G(2,4) \subset G(3,5)$.

Случаи, когда число переменных $n=2$ или коразмерность $p=1$, выделяются и в других ветвях математики. По нашему мнению это связано с афффинной классификацией точек многомерных поверхностей.

Например, при изучении эллиптических систем в частных производных случай одного уравнения и случай системы от двух неизвестных также выделяются из общей ситуации. Одно уравнение соответствует гиперповерхностям, система от двух переменных - двумерным поверхностям произвольной коразмерности.

\section{3. Сильно параболические подмногообразия}

3.1. Локальное строение сильно параболической поверхности в римановом пространстве. Для двумерных регулярных класса $C^{k}(k \geqslant 2)$ поверхностей в $\mathbb{E}^{3}$ определен класс развертьваюшихся поверхностей, все точки которых либо параболические, либо точки уплощения. Заметим, что ранг второй квадратичной формы этих поверхностей меньше или равен 1.

Через каждую точку развертывающейся поверхности проходит прямолинейный отрезок, вдоль которой касательная плоскость стационарная. Регулярная развертываюшаяся поверхность имеет нулевую гауссову кривизну. Регулярные поверхности нулевой гауссовой кривизны в $\mathbb{E}^{3}$ являются развертьваюшимися. Полная регулярная поверхность нулевой гауссовой кривизны в $\mathbb{E}^{3}$ является цилиндром. 
ТАБЛИЦА 2

\begin{tabular}{|c|c|c|c|}
\hline № & $\begin{array}{c}\text { Элементарные } \\
\text { делители }\end{array}$ & $\begin{array}{c}\text { Канонический вид } \\
\text { пучка вторых } \\
\text { квадратичных форм }\end{array}$ & $\begin{array}{c}\text { Главные кривизны } \\
\text { тензора Риччи }\end{array}$ \\
\hline $1 \mathrm{a}, 6$ & $\begin{array}{c}\lambda-\lambda_{1} ; \lambda-\lambda_{2} ; \lambda-\lambda_{3} \\
\lambda_{1} \neq \lambda_{2} \neq \lambda_{3} \neq \lambda_{1}\end{array}$ & $\pm x_{2}^{2}+x_{3}^{2} ; \pm x_{1}^{2}+x_{3}^{2}$ & $\begin{array}{c}0 \leqslant k_{1}, k_{2}<1 \\
0<k_{3}<2\end{array}$ \\
\hline $2 \mathrm{a}, \sigma$ & $\begin{array}{c}\lambda-\lambda_{1} ; \lambda-\lambda_{1} ; \lambda-\lambda_{3} \\
\lambda_{1} \neq \lambda_{3}\end{array}$ & $x_{3}^{2} ; \pm x_{1}^{2}+x_{2}^{2}$ & $\begin{array}{c}0 \leqslant k_{1}, k_{2}<1 \\
k_{3}=1\end{array}$ \\
\hline $\begin{array}{c}\text { За,б } \\
\text { вырожд. }\end{array}$ & $\lambda-\lambda_{1} ; \lambda-\lambda_{1} ; \lambda-\lambda_{1}$ & $0 ; x_{1}^{2}+x_{2}^{2} \pm x_{3}^{2}$ & $k_{1}=k_{2}=k_{3}=1$ \\
\hline 4 & $\begin{array}{c}\lambda-\lambda_{1} ; \lambda^{2}-2 \alpha \lambda+ \\
\left(\alpha^{2}+\beta^{2}\right) ; \beta \neq 0\end{array}$ & $\begin{array}{c}-x_{2}^{2}+2 x_{2} x_{3}+x_{3}^{2} \\
x_{1}^{2}+x_{2}^{2}+2 x_{2} x_{3}-x_{3}^{2}\end{array}$ & $\begin{array}{c}0 \leqslant k_{1}, k_{2} \leqslant 1 \\
1<k_{3} \leqslant 2\end{array}$ \\
\hline $5 \mathrm{a}, 6$ & $\begin{array}{c}\left(\lambda-\lambda_{1}\right)^{2} ; \lambda-\lambda_{3} \\
\lambda_{1} \neq \lambda_{3}\end{array}$ & $\begin{array}{c}x_{2}^{2} \pm x_{3}^{2} \\
2 x_{1} x_{2}-x_{2}^{2}\end{array}$ & $\begin{array}{c}0 \leqslant k_{1}, k_{2} \leqslant 1 \\
1 \leqslant k_{3} \leqslant 2\end{array}$ \\
\hline 6 & $\left(\lambda-\lambda_{1}\right)^{2} ; \lambda-\lambda_{1}$ & $x_{2}^{2} ; 2 x_{1} x_{2}+x_{3}^{2}$ & $\begin{array}{l}0 \leqslant k_{1}<1 \\
k_{2}=k_{3}=1\end{array}$ \\
\hline 7 & $\left(\lambda-\lambda_{1}\right)^{3}$ & $2 x_{2} x_{3} ; x_{2}^{2}+2 x_{1} x_{3}$ & $\begin{array}{c}0 \leqslant k_{1}, k_{2}<1 \\
1<k_{3}<2\end{array}$ \\
\hline 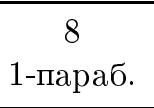 & $\begin{array}{c}\text { пучок } \\
\text { вырожден }\end{array}$ & $2 x_{2} x_{3} ; 2 x_{1} x_{3}$ & $\begin{array}{c}0 \leqslant k_{1} ; k_{2}<1 \\
k_{3}=1\end{array}$ \\
\hline
\end{tabular}

А.В. Погорелов обобщил эти результаты на класс гладких поверхностей ограниченной внешней кривизны в $\mathbb{E}^{3}[24]$.

Гладкую поверхность ограниченной внешней кривизны мы будем назьвать поверxностью нулевой кривизнь, если ее положительная и отрищательная кривизны равны нулю.

Поверхность нулевой кривизны развертьвающаяся (т.е. локально изометрична плоскости) и имеет обычное для регулярных развертьвающихся поверхностей строение. Полная поверхность с нулевой кривизной цилиндрическая [24].

На случай гладких гиперповерхностей эти результаты обобшены в [6].

В [6] доказано: если двумерная хаусдорфова мера сферического изображения гладкой класса $C^{1}$ гиперповерхности $F^{n-1}$ в евклидовом пространстве $\mathbb{E}^{n}$ равна нулю, то через каждую точку $F^{n-1}$ проходит $(n-2)$-образующая, вдоль которой касательная гиперплоскость стационарна.

Под образуюшей мы понимаем связную область на $(n-2)$-мерной плоскости.

Если гиперповерхность полная, то она является цилиндром с $(n-2)$-мерной образующей.

Для общих выпуклых гиперповерхностей аналогичные результаты доказаны, если $k$-мерная хаусдорфова мера сферического изображения равна нулю.

Сейчас мы введем класс регулярных подмногообразий произвольной коразмерности, которьй является обобшением класса развертьваюшихся поверхностей на много- 
мерньй случай.

Пусть $F^{l}$ - класса $C^{2}$ регулярное подмногообразие в римановом пространстве $M^{n}$, $Q \in F^{l}$ - точка на подмногообразии, $\xi$ - единичное нормальное векторное поле в окрестности точки $Q, X, Y$ - векторные поля, касательные к многообразию, $\bar{\nabla}$ - связность Леви-Чевита в римановом многообразии $M^{n}, \nabla$ - индуцированная связность на $F^{l}, \nabla^{\perp}$ - связность в нормальном расслоении $F^{l}, \alpha(X, Y)$ - векторная вторая квадратичная форма $F^{l}, A_{\xi}$ - линейное преобразование в $T_{Q} F^{l}$, соответствующее второй квадратичной форме относительно нормали $\xi$.

Тогда

$$
\begin{gathered}
\nabla_{\xi}=-A_{\xi} X+\nabla_{X}^{\perp} \xi \\
\bar{\nabla}_{X} Y=\nabla_{X} Y+\alpha(X, Y), \\
\left\langle A_{\xi} X, Y\right\rangle=\langle\alpha(X, Y), \xi\rangle,
\end{gathered}
$$

где $\langle\cdot, \cdot\rangle$ - скалярное произведение в $M^{n}$.

С. Черн и Н. Кейпер дали определение внешнего нуль-индекса подмногообразия [45].

ОПРЕДЕЛЕНИЕ. Внешний нуль-индекс $\mu(Q)$ точки $Q \in F^{l} \subset M^{n}$ есть максимальная размерность подпространства $L(Q)$ касательного пространства $T_{Q} F^{l}$ такого, что

$$
A_{\xi} y=0
$$

для любого вектора $y \in L(Q)$ и для любой нормали $\xi \in N_{Q} F^{l}$ в этой точке.

Подмногообразие назьвается $k$-сильно параболическим, если внешний нуль-индекс $\mu \geqslant k$ для каждой точки подмногообразия.

Регулярная поверхность нулевой гауссовой кривизны в $\mathbb{E}^{3}$ является 1-сильно параболической поверхностью. Если в окрестности точки $Q$ нуль-индекс $\mu$ постоянен, то на поверхности класса регулярности $C^{3}$ мы имеем дифференщируемое нуль-распределение $L(Q)$. Однако, без дополнительных требований для поверхностей в римановом пространстве это распределение может быть даже неинтегрируемым.

Пусть вдоль подмногообразия $F^{l}$ тензор кривизны объемлющего риманового многообраазия $M^{n}$ удовлетворяет условию:

$$
R(X, Y) Z^{\perp}=0
$$

где $R$ - оператор кривизны, $X, Y, Z$-векторы, касательные к $F^{l}, \perp$ - ортогональная проекция на нормальное пространство к $F^{l}$.

Для пространства постоянной кривизны $C$

$$
R(X, Y) Z=C(\langle X, Z\rangle Y-\langle Y, Z\rangle X)
$$

и условие (3.1.4) удовлетворяется вдоль произвольного подмногообразия. 
Лемма 3.1 .1 [55]. Пусть $F^{l}$ есть регулярное класса $C^{3}$ подмногообразие в римановом пространстве $M^{n}$. Предположим, что в окрестности точки $P_{0} \in F^{l}$ нуль-индекс $\mu(Q)=k=$ const $и$ в точках подмногообразия выполняется условие (3.1.4). Тогда нуль-распределение $L(Q)$ интегрируемо и слои $S L(Q)$ являются вполне геодезическими подмногообразиями обгемлющего пространства $M^{n}$.

Нормальное пространство стационарно вдоль слоев, т.е. произвольная нормаль $\xi(Q)$ в точке $Q$ слоя $S L(Q)$ после параллельного перенесения в связности обгемлющего пространства вдоль пути $\gamma \in S L(Q)$ остается нормалью к подмногообразию $F^{l}$.

Если $F^{l}$ есть полное подмногообразие и $\mu\left(P_{0}\right)=\min _{Q \in F^{l}} \mu(Q)=k$, то слой $S L\left(P_{0}\right)$ является полным вполне геодезическим подмногообразием.

Если обгемлющее пространство есть пространство постоянной кривизнь, то слои являются вполне геодезическими подмногообразиями постоянной кривизны: в случае евклидова пространства - больиими сферами, в случае пространства Лобачевского - пространствами Лобачевского меньшей размерносmu.

ДоКАЗАТЕЛЬСТВО лЕмМЫ 3.1.1. Пусть $y^{a}=y^{a}\left(u^{1}, \ldots, u^{l}\right)$ - регулярная параметризация $F^{l}$ в окрестности точки $P_{0}, y^{a}(a=1, \ldots, n)-$ координаты в $M^{n}, u^{i}$ $(i=1, \ldots, l)$ - координаты на $F^{l}$. Параметризацию поверхности мы можем выбрать так, чтобы в произвольной фиксированной точке $Q_{0}$ коэффициенты метрического тензора подмногообразия $F^{l} g_{i j}\left(Q_{0}\right)=\delta_{i j}$, где $\delta_{i j}$ - символ Кронекера, а символы Кристоффеля $\Gamma_{i j}^{k}\left(Q_{0}\right)=0$. Пусть $n_{\alpha}(\alpha=1, \ldots, n-l)$ - ортонормальньй базис нормалей в точке $Q_{0}$. Параллельно их перенесем в нормальной связности вдоль геодезических подмногообразия $F^{l}$, исходящих из точки $Q_{0}$.

Мы получим в окрестности точки $Q_{0}$ ортонормированньй базис нормальных векторных полей $n_{\alpha}$. Мы их обозначим тем же символом. Тогда любое нормальное векторное поле имеет вид $\xi=\xi^{\alpha} n_{\alpha}$ и ковариантная производная нормального векторного поля $\xi$ в нормальной связности представляется в следующей форме:

$$
\nabla_{X}^{\perp} \xi=\sum_{\beta} \mu_{\alpha \beta / i} \xi^{\alpha} x^{i} n_{\beta}
$$

где $X=\left(x^{1}, \ldots, x^{l}\right)$ - векторное поле, касательное к $F^{l}$. В силу выбора векторных полей $n_{\alpha}$ в точке $Q_{0}$ коэффициенты кручения $\mu_{\alpha \beta / i}=0$.

В обшем римановом пространстве в локальных координатах уравнения Кодацци имеют вид:

$$
A_{i j, k}^{\alpha}-A_{i k, j}^{\alpha}=\sum \mu_{\beta \alpha / k} A_{i j}^{\alpha}-\mu_{\beta \alpha / j} A_{i k}^{\alpha}+R_{a b c d} y_{i,}^{a} y_{j}^{c} y_{k}^{d} n_{\alpha}^{b}
$$

где $A_{i j}^{\alpha}$ - коэффициенты вторых квадратичных форм подмногообразия $F^{l}$ относительно нормали $n_{\alpha}, y_{i}^{a}=\frac{\partial y^{a}}{\partial u^{i}}, n_{\alpha}^{b}-$ координаты нормального векторного поля $n_{\alpha}$ [30].

В силу условия (3.1.4) и условий $g_{i j}\left(Q_{0}\right)=\delta i j, \Gamma_{i j}^{k}\left(Q_{0}\right)=0$ в точке $Q_{0}$ уравнения Кодацщи примут вид:

$$
\frac{\partial A_{i j}^{\alpha}}{\partial u^{k}}-\frac{\partial A_{i k}^{\alpha}}{\partial u^{j}}=0 .
$$


Пусть $X, Y$ - регулярные векторные поля, принадлежашие распределению $X(Q), Y(Q) \subset L(Q)$. Так как векторные поля $r_{i}=\frac{\partial r}{\partial u^{i}}$ образуют базис касательного пространства в каждой точке, где $r=\left\{y^{a}\left(u^{1}, \ldots, u^{l}\right)\right\}$ - регулярная параметризация подмногообразия, то

$$
X=x^{i} r_{i}, \quad Y=y^{i} r_{i}
$$

Так как $X, Y \subset L(Q)$, то из определения внешнего нуль-индекса следует

$$
A_{i j}^{\alpha} x^{j}=0 ; \quad A_{i j}^{\alpha} y^{j}=0
$$

для $\alpha=1, \ldots, n-l ; i=1, \ldots, l$.

Скобка Ли векторных полей $X, Y$ имеет вид

$$
[X, Y]=\left(\frac{\partial x^{i}}{\partial u^{s}} y^{s}-\frac{\partial y^{i}}{\partial u^{s}} x^{s}\right) r_{i}
$$

В точке $Q_{0}$ ковариантная производная имеет вид

$$
\nabla_{Y} X=\frac{\partial x^{i}}{\partial u^{s}} y^{s} r_{i}
$$

Мы хотим показать, что $\left.\nabla_{Y} X\right|_{Q_{0}} \subset L\left(Q_{0}\right)$.

Продифференцируем (3.1.6) по $u^{k}$. Мы получим

$$
\frac{\partial A_{i j}^{\alpha}}{\partial u^{k}} x^{i}+A_{i j}^{\alpha} \frac{\partial x^{i}}{\partial u^{k}}=0 .
$$

Умножив на $y^{k}$ и просуммировав по $k$, мы получим

$$
A_{i j}^{\alpha} \frac{\partial x^{i}}{\partial u^{k}} y^{k}=-\frac{\partial A_{i j}^{\alpha}}{\partial u^{k}} x^{i} y^{k} .
$$

Из (3.1.5), (3.1.6) и (3.1.9) мы получим следуюшее:

$$
-\frac{\partial A_{i j}^{\alpha}}{\partial u^{k}} x^{i} y^{k}=-\frac{\partial A_{i k}^{\alpha}}{\partial u^{j}} x^{i} y^{k}=A_{i k}^{\alpha} y^{k} \frac{\partial x^{i}}{\partial u^{j}}=0 .
$$

Из (3.1.3), (3.1.4), (3.1.9), (3.1.10) следует, что в точке $Q_{0} \nabla_{Y} X \subset L\left(Q_{0}\right)$.

Так как

$$
[X, Y]=\nabla_{Y} X-\nabla_{X} Y \subset L\left(Q_{0}\right),
$$

то из произвольности выбора точки $Q_{0}$ и теоремы Фробениуса следует, что дифференцируемое распределение $L(Q)$ интегрируемое. Из $\nabla_{Y} X \subset L(Q)$ и уравнения

$$
\nabla_{X} Y=\widetilde{\nabla}_{X} Y+h(X, Y)
$$

где $\widetilde{\nabla}$ - связность, индуцированная на слое, $h(X, Y)$ - вторая квадратичная форма слоя как подмногообразия $F^{l}$, следует, что

$$
h(X, Y)=0
$$


и слои являются вполне геодезическими подмногообразиями $F^{l}$.

Из определения нуль-индекса следует, что ограничения вторых квадратичных форм подмногообразия $F^{l}$ на слои тождественно равны нулю. Отсюда следует, что слои интегрируемого распределения являются вполне геодезическими подмногообразиями в объемлюшем пространстве. Перенесем параллельно в нормальной связности вдоль кривой слоя нормаль к слою в некоторой точке, $X$ - касательное векторное поле к кривой, $\xi$ - параллельное в нормальной связности векторное поле, $\nabla \frac{\perp}{X} \xi=0$. Векторное поле $\xi$ нормально к подмногообразию $F^{l}$,

$$
\bar{\nabla}_{X} \xi=-A_{\xi} X+\nabla_{X}^{\frac{1}{}} \xi .
$$

Так как $X \subset L(Q)$, то $A_{\xi} X=0$, и отсюда следует, что $\bar{\nabla}_{X} \xi=0$, и нормальное векторное поле $\xi$ параллельно в связности объемлющего пространства.

Пусть $\gamma$ - геодезическая линия слоя $S L(Q), Q$ - внутренняя точка слоя, $Q_{0} \in \gamma-$ граничная точка слоя, $X$ - вектор в точке $Q_{0}$, принадлежаший нуль-пространству. Допустим, что $\mu\left(Q_{0}\right)>\mu(Q)$. Введем координаты на $F^{l}$ такие, чтобы $u^{1}, \ldots, u^{k}$ были координатами слоя, $u^{1}$ - длина дуги на кривой $\gamma$. Выберем координаты в $F^{l}$ так, чтобы вдоль геодезической $g_{i j}=\delta_{i j} \Gamma_{i j}^{k}=0, \mu_{\alpha \beta / i}=0$. Параллельно перенесем вектор $X$ вдоль $\gamma$, мы получим векторное поле $X\left(u^{1}\right)$ со значением $X_{0}$ в точке $Q$.

В точке $Q_{0}$

$$
\begin{gathered}
A_{i j}^{\alpha} x^{j}=0 ; \\
\frac{\partial}{\partial u^{1}}\left(A_{i j}^{\alpha} x^{j}\right)=\frac{\partial A_{i j}^{\alpha}}{\partial u^{1}} x^{j}+A_{i j}^{\alpha} \frac{\partial x^{j}}{\partial u^{1}} .
\end{gathered}
$$

На подмногообразии $F^{l} A_{1 j}^{\alpha}=0$. Из уравнений Кодащци следует

$$
\nabla_{1}\left(A_{i j}^{\alpha} x^{j}\right)=\Gamma_{1 i}^{s} A_{s j}^{\alpha} x^{j} .
$$

Так как $A_{i j}^{\alpha} x^{j}=0$ в точке $Q_{0}$, то отсюда следует

$$
\frac{\partial}{\partial u^{1}}\left(A_{i j}^{\alpha} x^{j}\right)=0
$$

вдоль кривой $\gamma$, вектор $X_{0}$ принадлежит нуль-пространству в точке $Q$ и нуль-индекс $\mu\left(Q_{0}\right) \leqslant \mu(Q)$. Мы пришли к противоречию.

Если поверхность $F^{l}$ полная и нуль-индекс в точке $Q \in F^{l}$ минимален, т.е.

$$
\mu(Q)=\inf _{P \in F^{l}} \mu(P)=k,
$$

то в окрестности точки $Q$ нуль-индекс постоянен и равен $k$. Из предыдушего следует, что $\mu\left(Q_{0}\right)=k$ и точка $Q_{0}$ является внутренней точкой слоя $S L\left(Q_{0}\right)$, который в окрестности точки $Q_{0}$ совпадает со слоем $S L(Q)$. Отсюда следует, что любая геодезическая слоя неограниченно продолжаемая. Следовательно, слой $S L(Q)$ является полным вполне геодезическим подмногообразием.

В случае, когда объемлющее пространство является келеровым многообразием, а подмногообразие является келеровым подмногообразием, то слои являются вполне геодезическими келеровьми подмногообразиями [55]. 
3.2. Локальное строение сильно параболической поверхности в евклидовом пространстве. Пусть $F^{l}$ - регулярная поверхность в $E^{n}$ такая, что в окрестности точки $Q_{0} \in F^{l}$ нуль-индекс $\mu=k=$ const. Тогда через каждую точку поверхности проходит единственная плоскость $E^{k}(Q)$, вдоль которой касательное пространство стационарно. Через точку $Q_{0} \in F^{l}$ проведем плоскость $E^{n-k}$, перпендикулярную $E^{k}\left(Q_{0}\right)$. В окрестности точки $Q_{0}$ поверхность $F^{l-k}=F^{l} \cap E^{n-k}$ будет регулярной с радиус-вектором $\rho\left(u_{1}, \ldots, u_{l-k}\right)$, назовем ее базой $F^{l}$. Пусть $e_{l-k+1}, \ldots, e_{l}$ - ортонормированный базис $E_{k}\left(Q_{0}\right), S_{q}(Q)(q=l-k+1, \ldots, l)$ - векторы плоскости $E^{k}(Q)$ такие, что их ортогональная проекция на плоскость $E^{k}\left(Q_{0}\right)$ совпадает с $e_{q} ; v^{l-k+1}, \ldots, v^{l}$ - декартовы координаты в плоскости $E^{k}(Q)$ относительно базиса $S_{q}(Q)$. Тогда радиус-вектор поверхности $F^{l}$ в бесконечной полосе, содержащей образуюшую $E^{k}\left(Q_{0}\right)$, имеет вид

$$
r=\rho(u)+\sum_{q=l-k+1}^{l} S_{q}(u) v^{q}
$$

где $\rho_{i}=\frac{\partial \rho}{\partial u_{i}}(i=1, \ldots, l-k) ; N_{\alpha}(\alpha=1, \ldots, n-l=p)$ - ортогональньй базис нормалей к поверхности $F^{l-k} \subset E^{n-k}$. Так как векторы $e_{q}, \rho_{i}, N_{\alpha}$ в точках $Q \in$ $F^{l-k}$, близких к $Q_{0}$, составляют базис в $E^{n}$, то

$$
S_{q}=e_{q}+\sum c_{q}^{i} \rho_{i}+\sum \mu_{q}^{\alpha} N_{\alpha} .
$$

Так как $\frac{\partial r}{\partial v^{q}}=S_{q}$, то по формулам Вейнгартена

$$
\frac{\partial S_{q}}{\partial u_{i}}=\sum_{s=1}^{l-k} \Gamma_{i q}^{s} r_{s}+\sum_{t=l-k+1}^{l} \Gamma_{i q}^{t} S_{t}+\sum_{\alpha=1}^{p} A_{i q}^{\alpha} \nu_{\alpha},
$$

где $\nu_{\alpha}$ - нормали к поверхности $F^{l} ; \Gamma_{i q}^{s}, \Gamma_{i q}^{t}-$ коэффициенты Кристоффеля поверхности $F^{l}$. Из определения нуль-индекса $\mu$ и выбора системы координат следует, что $A_{i q}^{\alpha}=0$.

С другой стороны, из (3.2.2) получаем, что

$$
\frac{\partial S_{q}}{\partial u_{i}}=\frac{\partial c_{q}^{s}}{\partial u_{i}} \rho_{s}+\frac{\partial \mu_{q}^{\alpha}}{\partial u_{i}} N_{\alpha}+c_{q}^{s} \frac{\partial \rho_{s}}{\partial u_{i}}+\mu_{q}^{\alpha} \frac{\partial N_{\alpha}}{\partial u_{i}} .
$$

По формулам Вейнгартена для поверхности $F^{l-k} \subset E^{n-k}$

$$
\begin{aligned}
\frac{\partial \rho_{s}}{\partial u_{i}} & =\widetilde{\Gamma}_{s i}^{j} \rho_{j}+\Omega_{s i}^{\alpha} N_{\alpha} ; \\
\frac{\partial N_{\alpha}}{\partial u_{i}} & =-\Omega_{l i}^{\alpha} \widetilde{g}^{l m} \rho_{m}+\nu_{i \mid \alpha \beta} N_{\beta},
\end{aligned}
$$

где $\widetilde{g}_{i j}, \widetilde{g}^{i j}, \widetilde{\Gamma}_{s i}^{j} \widetilde{\Omega}_{l i}^{\alpha}, \nu_{i \mid \alpha \beta}$-ковариантные и контравариантные компоненты метрического тензора поверхности $F^{l-k}$, символы Кристоффеля этой метрики, коэффициенты 
второй квадратичной формы поверхности $F^{l-k}$ в $E^{n-k}$. Подставив (3.2.5) в (3.2.4), получим

$$
\begin{aligned}
\frac{\partial S_{q}}{\partial u_{i}}=\rho_{s}\left(\frac{\partial c_{q}^{s}}{\partial u_{i}}+\sum_{j=1}^{l-k} c_{q}^{j} \widetilde{\Gamma}_{i j}^{s}-\sum_{l=1}^{l-k} \sum_{\alpha} \mu_{q}^{\alpha} \Omega_{l i}^{\alpha} \widetilde{g}^{l s}\right) & \\
& +N_{\alpha}\left(\frac{\partial \mu_{q}^{\alpha}}{\partial u_{i}}+\sum_{s=1}^{l-k} c_{q}^{s} \Omega_{i s}^{\alpha}+\mu_{q}^{\beta} \nu_{i \mid \beta \alpha}\right) .
\end{aligned}
$$

Так как в каждой точке поверхности $F^{l}$ базисом $T_{Q} F^{l}$ являются как векторы $\left(r_{s}, S_{q}\right)$, так и $\left(\rho_{s}, S_{q}\right)$, то (3.2.3) переписьвается следующим образом:

$$
\frac{\partial S_{q}}{\partial u_{i}}=\sum_{s=1}^{l-k} B_{i q}^{s} \rho_{s}+\sum_{t=l-k+1}^{l} B_{i q}^{t} S_{t} .
$$

Сравнивая разложения $(3.2 .6)$ и $(3.2 .7)$, получаем $B_{i q}^{t}=0$. Поэтому

$$
\frac{\partial S_{q}}{\partial u_{i}}=B_{i q}^{s} \rho_{s}
$$

Так как $r_{s}=\rho_{s}+\frac{\partial S_{q}}{\partial u_{s}} v^{q}=\rho_{s}+B_{q s}^{i} \rho_{i} v^{q}, \frac{\partial r}{\partial v^{q}}=S^{q}$ - базис касательного пространства при всех значениях $v^{q}$, то матрица перехода от базиса касательного пространства $\rho_{s}, S_{q}$ к базису $r_{s}, S_{q}$ невырождена, т.е.

$$
\operatorname{det}\left(\delta_{i s}+B_{q s}^{i} v^{q}\right) \neq 0
$$

при любых значениях $v^{q}$.

Условия интегрируемости системы (3.2.8) с учетом (3.2.5) имеют следующий вид:

$$
B_{i q, j}^{s}=B_{j q, i}^{s}, \quad B_{i q}^{s} \Omega_{s j}^{\alpha}=B_{j q}^{s} \Omega_{s i}^{\alpha},
$$

где $j$ обозначает ковариантную производную по метрике поверхности $F^{l-k}$. Первое условие означает, что $B_{i q}^{s}=\frac{\partial g_{q}^{s}}{\partial u_{i}}$. Второе - что матрица $B_{q} \Omega^{\alpha}$ симметрична, здесь $B_{q}=\left(B_{i q}^{s}\right), \Omega^{\alpha}=\left(\Omega_{s i}^{\alpha}\right)$. На язьке внешних форм аналогичное условие встречается в статье М. А. Акивиса и В. В. Рыжкова [1]. Сильно параболические подмногообразия изучались также в работах М.А. Акивиса. В работе [23] изучалось локальное строение сильно параболических гильбертовых подмногообразий в гильбертовом пространстве. Доказано утверждение, аналогичное лемме 3.1.1.

В обшем случае при большой коразмерности сильно параболическое подмногообразие даже локально вырождается в конус или цилиндр.

Напомним, что точечная коразмерность р точки поверхности $F^{l} \subset E^{n}$ есть размерность линейного пространства вторых квадратичных форм поверхности в данной точке.

Э. Картан доказал, что если точечная коразмерность поверхности $p=l(l+1) / 2$, то сильно 1-параболическая поверхность есть конус или цилиндр [40]. В частности, отсюда следует теорема, доказанная в [3], так как в условиях теоремы $l=2, p=3$.

В. В. Рыжков доказал аналогичное утверждение при более слабом ограничении на точечную коразмерность: $p>l(l-1) / 2+1$ [26]. С учетом сказанного вьше этот результат можно переформулировать так: 
ТеОРема 3.2.1. Если в каждой точке регулярной поверхности $F^{l} \subset E^{n}$ точечная коразмерность $p>l(l-1) / 2+1$, то с точностью до параллельного переноса и гомотетии поверхность однозначно определяется по грассманову образу.

Вопрос о сушествовании сильно параболической поверхности с данной базой тесно связан с сопряженньми системами на поверхностях. Мы говорим, что ненулевые подпространства $T_{1}^{s}, T_{2}^{q} \subset T_{Q} F^{l}$ являются внешне сопряженными в точке $Q$, если $T_{Q} F^{l}=T_{1}^{s} \oplus T_{2}^{q}, s+q=l$, и для любых $X \in T_{1}, Y \in T_{2}$ будет

$$
\langle A(Q, \xi) X, Y\rangle=0
$$

где $\langle\cdot, \cdot\rangle$ - скалярное произведение в касательном пространстве, $A(Q, \xi)$ - матрица второй квадратичной формы относительно произвольной нормали $\xi$. Если базис $T_{Q} F^{l}$ выбрать так, чтобы $e_{1}, \ldots, e_{s} \in T_{1}^{s}, e_{s+1}, \ldots, e_{l} \in T_{2}^{q}$, то матрица второй квадратичной формы относительно произвольной нормали имеет вид:

$$
A=\left(\begin{array}{cc}
A_{1} & 0 \\
0 & A_{2}
\end{array}\right)
$$

где $A_{1}, A_{2}$ - квадратные матрицы порядка $s, q$ соответственно.

Заметим, что требование на точечную коразмерность $p>l(l-1) / 2+1$ гарантирует отсутствие в точках поверхности сопряженных подпространств.

В ситуации обшего положения при точечной коразмерности $p>l$ в точках поверхности нет сопряженных подпространств. Направление касательного пространства $X \in T_{Q} F^{l}$ назьвается асимптотическим, если $\langle A(Q, \xi) X, X\rangle=0$. Имеет место

ТеОРема 3.2.2. Пусть $F^{l}$ - регулярное подмногообразие в $E^{n}$, в точках которого нет сопряженных подпространств. Если 1) l нечетно; 2) на подмногообразии нет асимптотических направлений, то сильно параболическая поверхность $F^{l+1} \subset E^{n+1}$ с базой $F^{l}$ есть конус или иилиндр.

Для образуюшей большей коразмерности сильно $k$-параболическая поверхность будет либо цилиндром с $k$-мерной образуюшей, либо конусом с $(k-1)$-мерной вершиной.

Эквивалентной формулировкой этой теоремы является

ТЕОРема 3.2.2'. При условиях теоремы 3.2.2. подмногообразие $F^{l}$ однозначно с точностью до гомотетии и параллельного переноса определяется грассмановьлм образом.

Внешнее условие 2) можно заменить внутренним условием знакопостоянства секционной кривизны $F^{l}$.

Ненулевые подпространства $T_{1}^{s}, T_{2}^{q}$ касательного пространства $T_{Q} R^{l}$ риманова многообразия $R^{l}$ называются внутренне сопряженными в точке $Q$, если

$$
T_{Q} R^{l}=T_{1}^{s} \oplus T_{2}^{q}, \quad s+q=l,
$$

и для любых $Z, X \in T_{1} ; W, Y \in T_{2} ; T \in T_{Q} R^{l}$ будет

$$
\langle R(Z, X) T, Y\rangle=0 ; \quad\langle R(W, Y) T, X\rangle=0,
$$


где $R$ - тензор кривизны метрики $R^{l}$. Если базис $T_{Q} R^{l}$ выбрать так, чтобы $e_{1}, \ldots, e_{s} \in$ $T_{1}^{s}, e_{s+1}, \ldots, e_{l} \in T_{2}^{q}$, то в компонентах тензора кривизны условие сопряженности имеет вид:

$$
\begin{gathered}
R_{i j k \alpha}=R_{\alpha \beta \gamma i}=R_{i j \alpha \beta}=0, \\
i, j, k=1, \ldots, s ; \quad \alpha, \beta, \gamma=s+1, \ldots, l .
\end{gathered}
$$

Подпространства, сопряженные на поверхности $F^{l}$ относительно второй квадратичной формы, являются сопряженными относительно метрики $F^{l}$. Это непосредственно следует из формулы Гаусса. Поэтому условие отсутствия внешне сопряженных подпространств можно заменить на условие отсутствия внутренне сопряженных подпространств. И мы получим аналог теорем $3.2 .2,3.2 .2^{\prime}$, где условия теорем определяются только метрикой поверхности $F^{l}$. Поэтому существует внутренний аналог теоремы 3.2.2.

В случае четной размерности имеют место ТЕОРемы $3.2 .3,3.2 .3^{\prime}$, условия и утверждения которых совпадают с утверждениями теорем $3.2 .2,3.2 .2^{\prime}$ при дополнительном условии: 3) точечная коразмерность $p$ поверхности в каждой точке удовлетворяет неравенству $p>l(l+1) / 4$. Требование 2$)$ также можно заменить внутренним условием знакопостоянства секционной кривизны $F^{l}$. В случае положительной кривизны не нужно ограничение на точечную коразмерность.

В работе [15] рассматривались цилиндрические поверхности в пространстве Лобачевского, такие поверхности можно рассматривать и в сферическом пространстве. Это такие поверхности, которые при геодезическом отображении пространства постоянной кривизны в евклидово пространство переходят в конусы или цилиндры. Их можно описать, не обрашаясь к геодезическому отображению [15]. Теоремы 3.2.2, 3.2.3 при их переформулировке дают достаточные условия, когда сильно параболические поверхности в пространствах постоянной кривизны локально являются цилиндрами.

Пусть $F^{l}$ - регулярная поверхность в $E^{n}$ такая, что в окрестности точки $Q_{0} \in F^{l}$ нуль-индекс $\mu=k=$ const.

Рассмотрим случай $k=1$, в этом случае будет единственная матрица $B=\left(B_{j}^{s}\right)$. Выберем базис в точке $Q \in F^{l-k}$ базы $F_{l}$ так, чтобы матрица $B$ привелась к вешественному аналогу жордановой формы [5], т.е. имела вид:

$$
B=\left(\begin{array}{ccccc}
G\left(\mu_{1}, s_{1}\right) & & & & \\
& \ddots & & & \\
& & G\left(\mu_{q}, s_{q}\right) & & \\
& & T\left(\alpha_{1}, \beta_{1}, 2 r_{1}\right) & \\
& & & \ddots & \\
& & & & T\left(\alpha_{m}, \beta_{m}, 2 r_{m}\right)
\end{array}\right),
$$

где вдоль главной диагонали стоят непересекающиеся блоки, являющиеся либо жордановыми клетками порядка $s_{j}$ :

$$
G\left(\mu_{j}, s_{j}\right)=\left(\begin{array}{ccccc}
\mu_{j} & 1 & & 0 & \\
& \mu_{j} & 1 & & \\
& & \ddots & \ddots & \\
& & & \ddots & 1 \\
& 0 & & & \mu_{j}
\end{array}\right)
$$


либо вешественными аналогами жордановых клеток:

$$
T\left(\alpha_{j}, \beta_{j}, 2 r_{j}\right)=\left(\begin{array}{ccccccccc}
\alpha_{j} & \beta_{j} & 1 & 0 & & & & & 0 \\
-\beta_{j} & \alpha_{j} & 0 & 1 & 0 & & & & 0 \\
0 & 0 & \alpha_{j} & \beta_{j} & 1 & 0 & & & 0 \\
\vdots & \vdots & -\beta_{j} & \alpha_{j} & 0 & 1 & & & 0 \\
\vdots & \vdots & 0 & 0 & \ddots & \ddots & \ddots & & \vdots \\
& & & & \ddots & \ddots & \ddots & 1 & 0 \\
& & & & & \ddots & \ddots & 0 & 1 \\
& & & & & & \ddots & \alpha_{j} & \beta_{j} \\
0 & 0 & 0 & 0 & & & & -\beta_{j} & \alpha_{j}
\end{array}\right),
$$

где $\alpha_{j}+i \beta_{j}$ - комплексное собственное число матрицы $B, 2 r_{j}$ - порядок матрицы, $\beta_{j} \neq 0$.

ЛЕмма 3.2.1. Если матрица В имеет различные собственные значения, не считая комплексно сопряженных, то поверхность имеет сопряженные подпространства.

ЛЕмма 3.2.2. Если матрица $B$ имеет клетку, әде $s_{j}, r_{j}>1$, то базовая поверхность $F^{l}$ имеет асимптотическое направление, а также при $l>2$ есть двумерное направление, по которому секционная кривизна равна нулю.

ДокАЗАТЕЛЬство теоремы 3.2.2. Так как $l$ нечетно, то в каждой точке матрица $B$ имеет действительное собственное значение $\lambda(u)$. Из условия теоремы и леммы 3.2.1 следует, что все собственные значения матрищы $B$ совпадают с $\lambda(u)$. Из леммы 3.2.2 и условия отсутствия асимптотических направлений на поверхности $F^{l}$ следует, что для всех клеток $s_{j}=1$, матрица

$$
B=\lambda(u) E,
$$

где $E$ - единичная матрица, и матрица $B$ имеет этот вид в любой системе координат на поверхности $F^{l}$. Из тождества $(3.2 .10)$ следует, что $\lambda=$ const и поверхность является конусом при $\lambda \neq 0$ и цилиндром при $\lambda=0$.

Если размерность образуюшей $k>1$, то линейной комбинацией образующих мы получим, что матрица $B_{1}$ имеет вид (3.2.14), а остальные $B_{q}=0$, и тем самьм получим утверждение теоремы и в этом случае.

Знакопостоянство кривизны, как следует из леммы 3.2.2, также влечет отсутствие клеток, для которых $s_{j} \neq 1$.

При $l$ четном, если матрища $B$ имеет вещественные собственные значения, рассуждения не изменяются; если все собственные значения комплексные, то из лемм $3.2 .1,3.2 .2$ следует, что они совпадают и для клеток $T\left(\alpha_{j}, \beta_{j}, 2 r_{j}\right), r_{j}=1$, и 
матрица $B$ имеет вид:

$$
B=\left(\begin{array}{ccccccc}
\alpha & \beta & 0 & & & & \\
-\beta & \alpha & & & & & \\
& & \alpha & \beta & & 0 & \\
& & -\beta & \alpha & & & \\
& & & & \ddots & & \\
& & 0 & & & \alpha & \beta \\
& & & & & -\beta & \alpha
\end{array}\right),
$$

где $\lambda=\alpha+i \beta-$ собственные значения матрицы $B$. Из тождества (3.2.10) и вида матрищы $B$ следует, что матрица $\Omega^{\alpha}$ вторых квадратичных форм для всех нормалей имеет следуюший вид:

$$
\Omega^{\alpha}=\left(\begin{array}{ccclc}
\Omega_{11}^{\alpha} & \Omega_{12}^{\alpha} & \ldots & \Omega_{1, l-1}^{\alpha} & \Omega_{1 l}^{\alpha} \\
\Omega_{12}^{\alpha} & -\Omega_{11}^{\alpha} & \ldots & \Omega_{1 l}^{\alpha} & -\Omega_{1, l-1}^{\alpha} \\
& & \ddots & & \\
\Omega_{1, l-1}^{\alpha} & \Omega_{1 l}^{\alpha} & \ldots & \Omega_{l-1, l-1}^{\alpha} & \Omega_{l-1, l}^{\alpha} \\
\Omega_{1 l}^{\alpha} & -\Omega_{1, l-1}^{\alpha} & \ldots & \Omega_{l-1, l}^{\alpha} & -\Omega_{l-1, l-1}^{\alpha}
\end{array}\right) .
$$

Но в этом случае точечная коразмерность поверхности $p \leqslant l(l+1) / 4$, и в условиях теоремы 3.2.3 такого случая не может быть.

Так как в случае такого строения пространства вторых квадратичных форм кривизна поверхности по площадкам, натянутым на векторы $e_{2 i-1}, e_{2 i}$, неположительна, то в случае положительной кривизны поверхности этот случай исключается и нет необходимости в ограничении на точечную коразмерность.

ДокАЗАТЕЛЬСтво ЛЕммы 3.2.1. Не ограничивая общности, будем рассматривать случай, когда матрица $B$ состоит из двух жордановых клеток, отвечающих различным собственным значениям $\lambda, \mu$. Рассмотрим случай, когда $\lambda$-вешественное, $\mu=\alpha+i \beta-$ комплексное собственные числа. Для других случаев доказательство аналогично. Пусть матрица $B$ порядка $l$ состоит из матрицы $G(\lambda, s)$ порядка $s$ и матришы $T(\alpha, \beta, 2 r)$ порядка $2 r, s+2 r=l$. Рассмотрим матрицы $C=B \Omega$, где $\Omega$ - матрица второй квадратичной формы, относительно произвольной нормали $n$. По условию (3.2.10) она является симметричной. Найдем элементы $c_{k, l-1}=c_{l-1, k}, c_{k l}=c_{l k}$ этой матрицы:

$$
\lambda \Omega_{k l}=\alpha \Omega_{l-1, k}+\beta \Omega_{l k}, \quad \lambda \Omega_{k, l-1}=-\beta \Omega_{l-1, k}+\beta \Omega_{l k} .
$$

Эта однородная система имеет только нулевое решение

$$
\Omega_{l-1, k}=\Omega_{l k}=0 .
$$

Теперь найдем элементы $c_{k, l-3}=c_{l-3, k}, c_{k, l-2}=c_{l-2, k}$ :

$$
\begin{aligned}
& \lambda \Omega_{k, l-2}=-\beta \Omega_{l-3, k}+\alpha \Omega_{l-2, k}+\Omega_{l k}, \\
& \lambda \Omega_{k, l-3}=\alpha \Omega_{l-3, k}+\beta \Omega_{l-2, k}+\Omega_{l-1, k} .
\end{aligned}
$$

Так как из предыдушего $\Omega_{l k}=\Omega_{l-1, k}=0$, то $\Omega_{k, l-2}=\Omega_{k, l-3}=0$. Продолжая аналогичным образом, получим $\Omega_{k \alpha}=0, \alpha=k+1, \ldots, l$. Перейдя к другой строке и последовательно продолжая этот процесс, получим $\Omega_{i \alpha}=0 ; i=1, \ldots, k ; \alpha=k+$ $1, \ldots, l$. Это и значит, что подпространства $T_{1}, T_{2}$ натянуты на векторы $e_{1}, \ldots, e_{k} ;$ $e_{k+1}, \ldots, e_{l}$ - сопряженные. 
ДоКАЗАТЕЛЬСТВо ЛЕммы 3.2.2. Сначала рассмотрим случай, когда жорданова клетка порядка $k>1$ отвечает действительному корню $\lambda$, и пусть она является первой в матрице $B$. Вычислим элементы $c_{k-1, k}=c_{k, k-1}$ матрицы: $\lambda \Omega_{k, k-1}=$ $-\lambda \Omega_{k-1, k}+\Omega_{k k}$, откуда $\Omega_{k k}=0$ для произвольной нормали в данной точке. Если $k>2$, то вычислим элементы $c_{k, k-2}=c_{k-2, k}$. Получим $\lambda \Omega_{k, k-2}=$ $\lambda \Omega_{k-2, k}+\Omega_{k-1, k}$, откуда $\Omega_{k-1, k}=0$, и по формуле Гаусса секционная кривизна поверхности по двумерной плошадке, натянутой на базисные векторы $e_{k-1}, e_{k}$, равна нулю.

Если $l \geqslant 3$ и $k=2$, то найдется еще одна жорданова клетка порядка $s \geqslant 1$, отвечающая собственному значению $\mu$. Если $\mu \neq \lambda$, то из леммы 3.2.1 вытекает, что $\Omega_{k, k+s}=0$ вследствие сопряженности соответствуюших подпространств. Мы считаем, что клетка, отвечающая $\mu$, идет следом за клеткой, отвечающей $\lambda$. Если $\mu=\lambda$, то $c_{k+s, k-1}=c_{k-1, k+s}$. Получим $\lambda \Omega_{k+s, k-1}=\lambda \Omega_{k-1, k+s}+\Omega_{k, k+s}$. Откуда $\Omega_{k, k+s}=0$. Если $\lambda=\alpha+i \beta$, то проводим аналогичные вычисления и получаем сушествование двумерной асимптотической площадки.

3.3. Полные сильно параболические многомерные поверхности в евклидовом пространстве. Полные регулярные поверхности нулевой гауссовой кривизны в евклидовом пространстве $\mathbb{E}^{3}$ являются цилиндрами. Когда многомерныеполные сильно параболические подмногообразия в евклидовом пространстве являются цилиндрами? Без дополнительных требований на подмногообразие это не так. Поверхность $F^{3}$ в $\mathbb{E}^{4}$, заданная явно над трехмерной плоскостью в виде:

$$
z=x_{1} \cos x_{3}+x_{2} \sin x_{3}
$$

имеет в каждой точке нуль-индекс $\mu=1$, но не является цилиндром с одномерной образуюшей [60].

Ограничения могут быть как внешнегеометрические, так и связанные с кривизной метрики, индуцированной на подмногообразии.

ОПРЕДЕЛЕНИЕ. Пусть $r(Q, \xi)$ есть ранг второй квадратичной формы подмногообразия $F^{l}$ в римановом пространстве $M^{n}$ в точке $Q$ относительно нормали $\xi$. Рангом второй квадратичной формы поверхности $F^{l}$ в точке $Q$ назьвается целое неотрицательное число

$$
r(Q)=\max _{\xi \in N_{Q}} r(Q, \xi),
$$

где $N_{Q}$ - нормальное пространство в точке $Q$.

ОПРЕДЕЛЕНИЕ. Пусть вторая квадратичная форма $A(Q, \xi)$ после приведения к диагональному виду имеет $k_{1}$ положительных и $k_{2}$ отрицательных членов. Тогда

$$
j(Q, \xi)=\min \left(k_{1}, k_{2}\right)
$$

Типом точки $Q$ назьвается число

$$
j(Q)=\min j(Q, \xi)
$$

где минимум берется по всем нормалям в точке $Q$, для которых $r(Q, \xi)=r(Q)[7]$. 
Если $j(Q)=0$, то существует нормаль в точке, относительно которой вторая квадратичная форма является неотрицательно определенной. При этом, если ранг $r(Q)=l$, то относительно некоторой нормали вторая квадратичная форма строго определеннал. Если $r(Q)=l-k$, то относительно некоторой нормали ограничением второй квадратичной формы на $(l-k)$-мерную плоскость, ортогональную нуль-пространству, будет положительно определенная форма.

Поверхности с нулевым типом являются в некотором смысле обобщением выпуклых гиперповерхностей на подмногообразия произвольной коразмерности. Для полных подмногообразий справедлива

Теорема 3.3.1 [10], [7]. Пусть $F^{l}$ - полное регулярное связное подмногообразие в евклидовом пространстве $\mathbb{E}^{n}$ такое, что

1) нуль-индекс $\mu(Q)=k$ один и тот же во всех точках подмногообразия;

2) тип точки $j(Q)=0$ для всех точек поверхности.

Тогда подмногообразие $F^{l}$ есть иилиндр с $k$-мерной образующей.

ДокАЗАТЕЛЬСТво ТЕОРЕМЫ 3.3.1. Пусть $Q_{0} \in F^{l}$ - произвольная точка $F^{l}$. Через точку $Q_{0}$ проходит единственная плоскость $E^{k}\left(Q_{0}\right) \in F^{l}$, вдоль которой касательная плоскость стационарна. В окрестности плоскости $E^{k}\left(Q_{0}\right)$ радиус-вектор $F^{l}$ имеет вид

$$
r=\rho(u)+\sum_{q=l-k+1}^{l} S_{q}(u) \theta^{q},
$$

которьй задает регулярную параметризацию подмногообразия: $-\infty<\theta^{q}<+\infty$, $u^{1}, \ldots, u^{l-k}$ - регулярные координаты на $F^{l-k}$-ортогональном сечении подмногообразия плоскостью, перепендикулярной $E^{k}\left(Q_{0}\right)$.

В силу регулярности подмногообразия и полноты слоев неравенство (3.2.9) вьполняется при всех значениях $\theta^{q}$. Так как $j(Q)=0$, то существует единичное нормальное векторное поле $N_{1}$ вдоль ортогонального сечения $F^{l-k}$ в окрестности точки $Q_{0}$ такое, что вторая квадратичная форма $F^{l-k}$ относительно нормального векторного поля $N_{1}$ является положительно определенной.

Пусть $\Omega^{1}=\left(\Omega_{s j}^{1}\right)$ - матрица коэффициентов этой формы. Тогда из $(3.2 .9)$ следует, чTо

$$
\operatorname{det}\left(\Omega_{s j}^{1}+B_{q s}^{i} \Omega_{i j}^{1} \theta^{q}\right) \neq 0 .
$$

Из условия (3.2.10) следует, что матрища $\left(B_{q i}^{s} \Omega_{s j}^{1}\right)$ - симметрическая.

В силу полноты слоя и положительной определенности матрицы $\Omega^{1}$ вьполнение неравенства (3.3.1) возможно лишш при условии $B_{q s}^{i}=0$.

Из (3.2.8) следует, что

$$
\frac{\partial S_{q}}{\partial u^{i}}=0
$$

и $S_{q}=\bar{C}_{q}$ есть постоянньй вектор в окрестности точки $Q_{0}$. Отсюда следует, что в окрестности образуюшей $E^{k}\left(Q_{0}\right)$ подмногообразие $F^{l}$ является цилиндром с $k$-мерной образуюшей.

Из постоянства нуль-индекса и связности подмногообразия следует утверждение теоремы. 
ПРЕДПОЛОЖЕнИЕ. Теорема верна при условии $\mu(Q) \leqslant l-1$ без условия постоянства нуль-индекса.

Внешнегеометрическое условие $j(Q)=0$ следует из внутренних условий на кривизну Риччи подмногообразия. Справедлива

Лемма 3.3.1. Пусть подмногообразие $F^{l} \subset \mathbb{E}^{n}$ в точке $Q \in F^{l}$ имеет неотрицательную кривизну Риччи. Тогда тип точки

$$
j(Q)=0
$$

ДоКАЗАТЕЛЬСТВо ЛЕммы 3.3.1. Пусть $H$ есть вектор средней кривизны в точке $Q, \xi_{1}, \ldots, \xi_{n-l}-$ базис взаимноортогональных единичных нормальных векторов в точке $Q$, вектор $\xi_{1}$ имеет направление вектора $H$. Выберем в касательном пространстве $T_{Q} F^{l}$ базис, состояший из взаимно ортогональных единичных собственных векторов второй квадратичной формы $A^{1}$ относительно нормали $\xi_{1} ; e=\left(e_{1}, \ldots, e_{l}\right)-$ этот базис.

Секционная кривизна в направлении площадки $\left\{e_{i}, e_{j}\right\}, i \neq j$, вычисляется по формуле

$$
k\left(e_{i}, e_{j}\right)=A^{1}\left(e_{i}, e_{i}\right) A^{1}\left(e_{j}, e_{j}\right)+\sum_{\alpha=2}^{n-1}\left[A^{\alpha}\left(e_{i}, e_{i}\right) A^{\alpha}\left(e_{j}, e_{j}\right)-\left(A^{\alpha}\left(e_{i}, e_{j}\right)\right)^{2}\right] .
$$

Это следует из уравнений Гаусса.

Кривизна Риччи в направлении вектора $e_{i}$

$$
\begin{aligned}
\operatorname{Ric}\left(e_{i}\right)=A^{1}\left(e_{i}, e_{i}\right) \sum_{j \neq i}^{n} & A^{1}\left(e_{j}, e_{j}\right) \\
& +\sum_{\alpha=2}^{n-l} A^{\alpha}\left(e_{i}, e_{i}\right)\left[\sum_{j \neq i}^{n} A^{\alpha}\left(e_{j}, e_{j}\right)\right]-\sum_{\alpha=2}^{n-l} \sum_{j=1}\left(A^{\alpha}\left(e_{i}, e_{j}\right)\right)^{2} .
\end{aligned}
$$

В силу выбора нормалей в точке $Q$

$$
\sum_{j \neq i}^{n} A^{\alpha}\left(e_{j}, e_{j}\right)=-A^{\alpha}\left(e_{i}, e_{i}\right), \quad \alpha \neq 1
$$

и кривизна Риччи

$$
\operatorname{Ric}\left(e_{i}\right)=A^{1}\left(e_{i}, e_{i}\right)\left(|H|-A^{1}\left(e_{i}, e_{i}\right)\right)-\sum_{\alpha=2}^{n-l}\left(A^{\alpha}\left(e_{i}, e_{i}\right)\right)^{2}-\sum_{\alpha=2}^{n-l} \sum_{j \neq i}\left(A^{\alpha}\left(e_{i}, e_{j}\right)\right)^{2},
$$

где $|H|$ - модуль средней кривизны в точке $Q$.

Так как Ric $\geqslant 0$, то

$$
A^{1}\left(e_{i}, e_{i}\right)\left(|H|-A^{1}\left(e_{i}, e_{i}\right)\right) \geqslant 0
$$

для всех $i=1, \ldots, l$. 
Отсюда следует, что $A^{1}\left(e_{i}, e_{i}\right) \geqslant 0$ и вторая квадратичная форма относительно нормали $\xi_{1}$ неотрицательно определенная. Если кривизна Риччи неотрицательна и $A^{1}\left(e_{i}, e_{i}\right)=0$, то

$$
A^{\alpha}\left(e_{i}, e_{i}\right)=0, \quad A^{\alpha}\left(e_{i}, e_{j}\right)=0, \quad \alpha=2, \ldots, n-l, \quad j \neq i,
$$

и вектор $e_{i}$ принадлежит нуль-пространству. Отсюда следует, что $r\left(Q, \xi_{1}\right)=r(Q)$ и $j(Q)=0$.

Близкое утверждение в случае положительной секционной кривизны имеется в [20] .

Лемма 3.3.1 справедлива и для подмногообразия риманова пространства, если мы будем рассматривать внешнюю кривизну Риччи, которую мы определим следующим образом: возьмем ограничение тензора кривизны $\bar{R}$ объемлющего пространства на касательную плоскость $T_{Q} F^{l}$ подмногообразия и кривизну Риччи в направлении вектора $e \in T_{Q} F^{l}$, определенную таким образом:

$$
\widetilde{\operatorname{Ric}(e)}=\sum_{i=2}^{l} k\left(e, e_{i}\right),
$$

где $\left(e, e_{2}, \ldots, e_{l}\right)$ - ортогональный базис $T_{Q} F^{l}, k\left(e, e_{i}\right)$ - секционная кривизна объемлющего многообразия в направлении плошадки $\left\{e, e_{i}\right\}$.

Внешняя кривизна Риччи

$$
\overline{\operatorname{Ric}}(e)=\widetilde{\operatorname{Ric}}(e)-\operatorname{Ric}(e),
$$

где $\operatorname{Ric}(e)$ - кривизна Риччи в метрике подмногообразия $F^{l} . \operatorname{Ecли~} \operatorname{Ric}(e) \geqslant 0$, то лемма 3.3.1 имеет место в римановом пространстве. Если объемлющее многообразие стандартная сфера $S^{n}$ кривизны единица, то внешняя кривизна Риччи $\overline{\operatorname{Ric}}(e) \geqslant 0$ в случае, когда кривизна Риччи подмногообразия $F^{l}$ больше или равна $l-1$.

Из леммы 3.3.1 следует, что теорема 3.3.1 будет верна, если внешнее условие $j(Q)=0$ заменить условием неотрищательности кривизны Риччи.

ОПреДЕЛЕНИЕ. Пусть $M^{n}$ некомпактное полное риманово многообразие. Кривая $\gamma(s) \in M^{n}(-\infty<s<\infty)$, где $s$ - параметр длины дуги на $\gamma$, называется $п р я$ яой, если она является кратчайшей на каждом своем отрезке.

В. А. Топоногов доказал: если на полном некомпактном римановом многообразии $M^{n}$ неотрицательной секционной кривизны существует $k$ линейно независимых прямых, то $M^{n}$ есть риманово произведение $M^{n}=M^{n-k} \times E^{k}$, где $E^{k}$ изометрично евклидову пространству, $M^{n-k}-$ полное риманово многообразие неотрицательной секционной кривизны [27].

На случай неотрицательной кривизны Риччи теорема была обобщена Чигером и Громоллом [43].

Во внешней геометрии имеет место

ЛЕМма 3.3.2 [8]. Пусть подмногообразие $F^{l}$ является класса $C^{0}$ изометрическим погружением полного риманового многообразия $M^{l}$, которое является метрическим произведением $M^{l}=M^{l-k} \times E^{k}$, где $E^{k}-$ евклидов фактор.

Если образ $\left(Q_{0} \times E^{k}\right)$, әде $Q_{0}$ - фиксированная точка $M^{l-k}$, является $k$-мерной плоскостью евклидова пространства, то подмногообразие $F^{l}$ является иилиндром с k-мерной образующей. 
Лемма 3.3.3. Пусть $F$ - двумерная класса $C^{0}$ поверхность в евклидовом пространстве $E^{n}$, изометричная плоскости, и на $F$ лежит прямая р облемлющего пространства. Тогда F будет иилиндром с образующей, параллельной прямой $р$.

ДоКАЗАТЕЛЬСТво ЛЕммЫ 3.3.3. Пусть $\bar{F}$ - плоскость, изометричная поверхности $F ; \bar{Q} \in \bar{F}$ - точка, соответствуюшая $Q \in F$ по изометрии; $\overline{P Q}$ - расстояние на $\bar{F}$ между точками $\bar{P}, \bar{Q} ; P Q-$ расстояние между точками $P, Q$ в $E^{n}$.

Введем на $\bar{p}$ в качестве параметра длину $s$, отсчитываемую от фиксированной точки $\bar{P}(0) \in \bar{p} . \quad M(s)-$ кратчайшая, перпендикулярная $\bar{p}$, которая проходит через точку $\bar{P}(s) \in \bar{p}$.

Теперь через каждую точку $P(s) \in p$ поверхности $F$ проведем гиперплоскость $E^{n-1}(s)$, ортогональную $p, F(s)=E^{n-1}(s) \cap F$. Покажем, что $F(s)=M(s)$ для любого $s$. Допустим, что для какого-то $s_{0}$ в $F\left(s_{0}\right)$ будет содержаться точка $Q \in M\left(s_{1}\right)$, где $s_{1} \neq s_{0}$. Возьмем на $\bar{F}$ треугольник $\bar{P}\left(s_{0}\right) \overline{Q P}(s)$. Так как $\bar{Q} \notin \bar{M}\left(s_{0}\right)$, то кратчайшая $\bar{P}\left(s_{0}\right) \bar{Q}$ не перпендикулярна к $\bar{p}$. Точку $\bar{P}(s)$ будем выбирать на луче, которьй образует с $\bar{P}\left(s_{0}\right) \bar{Q}$ острый угол.

При достаточно большом $s$ угол $\bar{P}\left(s_{0}\right) \overline{Q P}(s)$ будет тупым и

$$
\bar{P}\left(s_{0}\right) \bar{P}(s)>\overline{Q P}(s) \text {. }
$$

Рассмотрим треугольник $P\left(s_{0}\right) Q P(s)$. Он прямоугольный с прямым углом $Q P\left(s_{0}\right) P(s)$.

Значит,

$$
P(s) Q>P\left(s_{0}\right) P(s)
$$

HO

$$
P\left(s_{0}\right) P(s)=\bar{P}\left(s_{0}\right) \bar{P}(s), \quad Q P(s) \leqslant \overline{Q P}(s) .
$$

Отсюда $\bar{P}(s) \bar{Q}>\bar{P}\left(s_{0}\right) \bar{P}(s)$. В результате приходим к противоречию, т.е.

$$
F(s)=M(s) .
$$

Пусть $\bar{Q}$ - произвольная точка $\bar{F} ; \bar{q}$ - прямая, проходящая через $\bar{Q}$ и параллельная $\bar{p}$. Покажем, что $q$ будет прямой евклидова пространства, параллельной $p$. Пусть $\bar{Q}_{1}, \bar{Q}_{2}$ - произвольные точки на $\bar{q}$. Опустим из $\bar{Q}_{1}, \bar{Q}_{2}$ перпендикуляры на $\bar{p} . \bar{P}_{1}, \bar{P}_{2}-$ основания перпендикуляров $\bar{P}_{1} \bar{P}_{2}=\bar{Q}_{1} \bar{Q}_{2}$.

Рассмотрим пространственньй четырехугольник $P_{1} P_{2} Q_{2} Q_{1}$. Натянем на этот четырехугольник евклидово пространство $E^{3}$. Началом прямоугольной системы координат в $E^{3}$ возьмем $P_{1}$, ось $z$ направим по прямой $p$. Точки $P_{1}, P_{2}, Q_{1}, Q_{2}$ имеют следуюшие координаты:

$$
\begin{gathered}
P_{1}(0,0,0), \quad P_{2}\left(0,0, z_{2}\right), \quad Q_{1}\left(x_{1}, y_{1}, 0\right), \quad Q_{2}\left(x_{2}, y_{2}, z_{2}\right), \\
P_{1} P_{2}=\left|z_{2}\right|, \quad Q_{1} Q_{2}=\sqrt{\left(x_{1}-x_{2}\right)^{2}+\left(y_{1}-y_{2}\right)^{2}+z_{2}^{2}} \geqslant P_{1} P_{2},
\end{gathered}
$$

но $P_{1} P_{2}=\bar{P}_{1} \bar{P}_{2}, Q_{1} Q_{2} \leqslant \bar{Q}_{1} \bar{Q}_{2}$ и, значит, $Q_{1} Q_{2} \leqslant P_{1} P_{2}$.

Отсюда $Q_{1} Q_{2}=P_{1} P_{2}$. А это возможно, если $x_{1}=x_{2}, y_{1}=y_{2}$, т.е. отрезок $Q_{1} Q_{2}$ будет параллелен $p$ и он будет кратчайшей на $\bar{F}$.

Ввиду произвольности точек $\bar{Q}_{1}, \bar{Q}_{2}, \bar{Q}$ утверждение леммы доказано.

Лемма 3.3.2 непосредственно следует из леммы 3.3.3. 
ТЕОРема 3.3.2. Пусть $F^{l}$ - полное регулярное класса $C^{3}$ подмногообразие в евклидовом пространстве $E^{n}$. Пусть во всех точках подмногообразия выполняются следующие условия:

1) нуль-индекс $\mu(Q) \geqslant k$;

2) кривизна Риччи неотрицательна.

Тогда подмногообразие $F^{l}$ является иилиндром $с k$-мерной образующей.

ДокАЗАТЕЛЬСТво ТеоремЫ 3.3.2. Пусть точка $Q_{0} \in F^{l}$ такова, что

$$
\mu\left(Q_{0}\right)=\min _{Q \in F^{l}} \mu(Q)
$$

Тогда из леммы 3.3 .1 следует, что через точку $Q_{0}$ проходит плоскость $E^{k}$, которая лежит на подмногообразии. Из теоремы, доказанной Чигером и Громоллом [43], следует, что с точки зрения внутренней геометрии $F^{l}$ есть метрическое произведение: $F^{l}=F^{l-k} \times E^{k}$, где $E^{k}$ - евклидов фактор. Из леммы 3.3 .2 следует, что $F^{l}$ является цилиндром с $k$-мерной образующей.

При условии неотрицательности секционной кривизны теорема была доказана $\Phi$. Хартманом [41]; для полных гиперповерхностей нулевой секционной кривизны Хартманом и Ниренбергом [42], так как в этом случае $\mu \geqslant l-1$; для гиперповерхностей неотрицательной секционной кривизны теорема доказана Сакстедером [60].

Для полных подмногообразий неположительной секционной кривизны в $E^{n}$ Л. Флорит доказал теорему о расшеплении этих подмногообразий. Мы говорим, что изометрическое погружение $g: M^{l} \rightarrow E^{l+p}$ расшепляется в $s$-произведение $(s \geqslant 2)$, если сушествует изометрическое погружение $g_{j}: M_{j}^{l_{j}} \rightarrow E^{l_{j}+p_{j}}, l_{j} \geqslant 1$, такое, что

$$
M^{l}=M_{1}^{l_{1}} \times \cdots \times M_{s}^{l_{s}} \text { и } g=g_{1} \times \cdots \times g_{s} .
$$

Теорема 3.3 .3 [52]. Пусть $F^{l}$ есть полное регулярное подмногообразие неположительной секционной кривизны с плоской нормальной связностью в евклидовом пространстве $E^{l+p}$. Предположим, что нуль-индекс во всех точках удовлетворяет неравенству

$$
\mu \leqslant l-p-s
$$

где $s, 2 \leqslant s \leqslant p,-$ челое число.

Тогда существует открытое плотное подмножество $U \subset F^{l}$ такое, что локально U есть s-произведение нигде не плоских подмногообразий.

Без ограничений на внутреннюю или внешнюю геометрию полная $k$-параболическая поверхность не является цилиндром с $k$-мерной образующей.

Для полных подмногообразий с постоянньм нуль-индексом $\mu=k$ мы определим сильный нуль-индекс $\mu_{c}(Q)$, равньй размерности линейного подпространства, натянутого на матрищы $B_{q}$. 
ОПРЕДЕЛЕНИЕ. Направление $x \in T_{Q} F^{l}$ называется асимптотическим, если для всех нормалей $\langle A(Q, \xi) x, x\rangle=0$, где $\langle\cdot, \cdot\rangle$ - скалярное произведение. Плоскость в $S \subset T_{Q} F^{l}$, все направления которой являются асимптотическими, назьвается асимптотической в точке $Q$. Максимальная размерность асимптотической плоскости называется индексом асимптотичности и обозначается $a(Q)$.

В общем случае $a(Q) \geqslant \mu(Q)$, так как все направления из нуль-пространства $L(Q)$ являются асимптотическими. В случае равенства $a(Q)=\mu(Q)$ справедлива

ТеОрема 3.3.4 [13]. Пусть $F^{l}$ есть полное регулярное подмногообразие в $E^{n}$, для которого сильный нуль-индекс постоянен, а нуль-индекс $\mu=l-2(l-3)$. Eсли индекс асимптотичности $a(Q)=\mu(Q)$, то подмногообразие $F^{l}$ является цилиндром $c(l-3)-u(l-5)$-мерньми образующими соответственно.

ЗАмЕчАнИЕ. Если тип $j(Q)$ - постоянный на подмногообразии, то при постоянном нуль-индексе $\mu=k$ в условиях теоремы 3.3.4 подмногообразие является цилиндром с $(k-j(r-j))$-мерными образующими, $j \leqslant\left[\frac{r}{2}\right] \leqslant 1$ и $r=2(3)$. Отсюда $j(r-j) \leqslant 1(2)$.

Кажется вполне вероятньм, что при $a(Q)=\mu(Q)$ должна сушествовать лучшая оценка размерности образующей цилиндра. Нам кажется, что при естественных предположениях размерность образующей цилиндра должна равняться $\mu=k$.

Для гиперповерхностей М. Дайшер и Д. Громолл доказали следуюшие теоремы.

ТеОРема 3.3 .5 [47]. Пусть $F^{n}$ есть полная регулярная гиперповерхность в $E^{n+l}$. Предположимм, что

1) нуль-индекс $\mu(Q)=n-2$ во всех точках поверхности;

2) средняя кривизна не меняет знак.

Тогда $F^{n}$ есть иилиндр с $(n-3)$-мерными образующими.

Если нуль-индекс $\mu(Q) \leqslant n-2$ на полной гиперповерхности $F^{l} \subset E^{n+1}$, то строение этих гиперповерхностей изучено в [48]. Эти результаты применены для изучения вопросов однозначности изометрического погружения гиперповерхностей.

Для келеровых подмногообразий в комплексном евклидовом пространстве имеет место обобшение теоремы Хартмана-Ниренберга.

ТЕОРема 3.3.6 [32]. Пусть $F^{l}(l-$ - комплексная размерность) есть полная келерова гиперповерхность в комплексном евклидовом пространстве $\mathbb{C}^{l+1}$.

Если нуль-индекс $\mu \leqslant 2$ во всех точках подмногообразия, то $F^{l}$ есть иилиндр, образующей которого является $(l-1)$-мерная комплексная плоскость, а направляющей - комплексная кривая $F^{1}$ в комплексном евклидовом пространстве $\mathbb{C}^{2}$.

Розенталь доказал общую теорему о цилиндричности келеровых подмногообразий B $\mathbb{C}^{n}$.

ТЕОРема 3.3 .7 [59]. Пусть $F^{l}$ есть полное, связное и односвязное келерово подмногообразие в $\mathbb{C}^{n}$ с постоянным нуль-индексом $\mu=k$.

Если голоморфная кривизна по всем голоморфнымм площадкам, ортогональнымм нуль-пространству, строго отричательна, то подмногообразие $F^{l}$ есть uилиндр с $k / 2$-мерной комплексной образующей. 
Теорема верна, если $\mu \geqslant k$ и выполнено внешнегеометрическое требование $a(Q)=\mu(Q)[17]$. Это условие эквивалентно требованию строгой отрицательности голоморфной кривизны по плошадкам, ортогональным нуль-пространству.

3.4. Вполне геодезичность полных сильно параболических подмногообразий в сферическом пространстве. Если в евклидовом пространстве мы находили условия, при которых сильно параболические подмногообразия являются цилиндрами, то аналогичной задачей в сферическом пространстве является задача нахождения условий вполне геодезичности. Имеет место

ТЕОРема 3.4.1. Пусть $F^{l}$ есть полное регулярное подмногообразие в сферическом пространстве $S^{n}$. Если нуль-индекс

1) $\mu(Q) \geqslant k(k>0)$ во всех точках подмногообразия;

2) $j(Q)=0$ хотя бь в одной точке, где нуль-индекс минимален, то подмногообразие $F^{l}$ является вполне геодезической большой сферой $S^{l}$ в $S^{n}$.

ДоКАЗАТЕЛЬСТВо ТЕОРемЫ 3.4.1. Пусть точка $Q_{0} \in F^{l}$ такова, что $\mu(Q)=k$, $j(Q)=0, U-$ окрестность, в которой $\mu(Q)=k=$ const. Из леммы 3.1 .1 следует, что через точки $Q \in U$ проходят полные слои вполне геодезические сферы $S^{k}(Q)$. Возьмем открытую полусферу сферы $S^{n}$ с центром $Q_{0}$. Осушествим геодезическое отображение этой полусферы на касательное в точке $Q_{0}$ евклидово пространство. Пусть $\bar{F}^{l}$ - область на поверхности $F^{l}$, образованная слоями, которые проходят через точки $Q \in U$. Ее образом при геодезическом отображении будет сильно параболическая поверхность $\widetilde{F}^{l}$ в евклидовом пространстве с $\mu=k ; j=0$ и полная вдоль слоев, которые являются образами вполне геодезических открытых полусфер [9]. В силу полноты вдоль слоев мы можем применить теорему 3.3.1, и $\widetilde{F}^{l}$ является цилиндром с $k$-мерной образуюшей. Но прообразом параллельных плоскостей являются вполне геодезические полусферы, которые пересекаются на гранище. Эти вполне геодезические полусферы лежат на вполне геодезических сферах, которые как слои слоения не пересекаются. Мы пришли к противоречию. Отсюда следует, что $\mu=l$, и подмногообразие $F^{l}$ является вполне геодезической большой сферой $S^{l}$.

Используя аналог леммы 3.3.1 для риманова подмногообразия, мы получаем

СлЕДСТВИЕ. Пусть $F^{l}$ есть полное регулярное подмногообразие в сферическом пространстве $S^{n}$. Если нуль-индекс $\mu(Q) \geqslant k$ во всех точках подмногообразия и кривизна Риччи $\operatorname{Ric}\left(F^{l}\right) \geqslant l-1$, то $F^{l}$ является вполне геодезической большой сферой $S^{l}$ в $S^{n}[34]$.

Д. Громолл и М. Дайшер [46] доказали аналогичньй результат лишш при условии, что кривизна Риччи больше или равна $l-1$ в некоторой точке, где нуль-индекс $\mu$ минимален. Снова в окрестности этой точки $j(Q)=0$, и в дальнейшем применимы все рассуждения теоремы 3.4.1.

Без всяких ограничений на кривизну подмногообразия в [46] показано, что в точках, где нуль-индекс минимален, сигнатура второй квадратичной формы относительно произвольной нормали равна нулю. То есть, число положительных и число отрицательных главных кривизн равны между собой и $j(Q)=\frac{l-\mu}{2}$. Действительно, из 
леммы 3.1.1 следует, что окрестность $U$ точки $Q$ расслаивается на полные вполне геодезические большие сферы, и центральная симметрия $I=-\mathrm{id}$ является инволюцией на $U$. Пусть $Q \in U$ - фиксированная точка, $\xi$ - нормаль в $Q$ и $S L(Q)$ - слой, проходяший через точку $Q$. После параллельного переноса нормали $\xi$ вдоль слоя она остается нормалью к поверхности. Из свойств инволюции $I$ следует, что

$$
I_{*} \circ A_{\xi(Q)}=A_{I_{*} \xi} \circ I_{*},
$$

где $I_{*}$ - индуцированное отображение касательных пространств, $A_{\xi}$ - оператор второй квадратичной формы.

Отсюда

$$
A_{\xi(Q)}=A_{-\xi(-Q)}=-A_{\xi(-Q)}
$$

Нормаль $\xi(-Q)$ получается параллельным переносом нормали $\xi(Q)$ вдоль слоя. При параллельном переносе нормали ранг второй квадратичной формы остается постоянным [46]. Из этого факта и равенства (3.4.1) следует, что число положительных главных кривизн равно числу отрицательных главных кривизн.

Д. Ферус изучал геодезические слоения на римановых многообразиях и дал оценку размерности слоя [50]. Полные сильно параболические подмногообразия в сферическом пространстве удовлетворяют условиям этой теоремы. Отсюда следует условие вполне геодезичности этих подмногообразий. Введем некоторые определения.

Пусть $\rho(l)-1$ есть максимальное число линейно независимых векторных полей на cфpepe $S^{l-1}$.

$$
\rho\left((2 k+1) 2^{c+4 d}\right)=2^{c}+8 d, \quad \text { где } 0 \leqslant c \leqslant 3 .
$$

Тогда $\nu(l)$ есть максимальное целое число, удовлетворяющее неравенству

$$
\rho(l-\nu(l)) \geqslant \nu(l)-1 .
$$

Число $\nu(l)$ имеет порядок $\log _{2} l$. Мы имеем $\nu(l)=l-($ наивысшая степень $2 \leqslant l$ ) для $l \leqslant 24$ и и $\nu(l)=0$ для $l=2^{k}$, или $l=16 q$, где $q \leqslant 16^{3}$ и $q \neq 1(\bmod 16)$.

Кроме того, $\nu(l) \leqslant \frac{1}{2}(l-1)$ и $\nu(l) \leqslant 8 d-1$ для $l \leqslant 16^{d}$, где $d$-любое положительное целое число.

ТЕОРема 3.4 .2 [50]. Пусть $M^{n}$ есть риманово многообразие и $T_{0}-\nu$-мерное слоение, удовлетворяющее следующим условиям:

1) слои являются полными вполне геодезическими подмногообразиями;

2) секчионная кривизна $M$ имеет постоянное положительное значение для всех смешанных площадок, натянутых на векторы $X u Y$, где $X \in T_{0}, a$ $Y \in T_{0}^{\perp}-$ ортогональный $\kappa$ слою вектор.

Eсли $\nu>\nu_{n}$, mo $\nu=n$.

Отсюда следует

ТеОРема 3.4.3. Пусть $F^{l}$ - полное регулярное подмногообразие сферического пространства $S^{n}$.

Если нуль-индекс $\mu(Q)>\nu(l)$ во всех точках подмногообразия $F^{l}$, то $\mu(Q)=l$ и подмногообразие $F^{l}$ есть стандартная вполне геодезическая сфера. 
ДоКАЗАТЕЛЬСТВО ТЕОРемЫ 3.4.3. Пусть точка $Q_{0} \in F^{l}$ такова, что

$$
\mu\left(Q_{0}\right)=\min _{Q \in F^{l}} \mu(Q)=\mu_{0}
$$

Тогда $\mu(Q)=\mu_{0}=$ const в некоторой окрестности $U$. Из леммы 3.1 .1 следует, что через каждую точку $Q \in U$ проходит полньй вполне геодезический слой $S L(Q)$. Из формулы Гаусса следует, что секционная кривизна в направлении смешанных площадок постоянна и равна кривизне объемлюшего пространства. Мы получаем слоение, удовлетворяющее условиям предыдущей теоремы.

ОПРеДЕЛЕНИЕ. Пусть $X, Y$-векторные поля, где $X \in T_{0}, Y \in T_{0}^{\perp} . P: T M \rightarrow T_{0}^{\perp}$ - ортогональная проекция. Тогда конулевой оператор $C$ определяется следуюшим образом:

$$
C_{X} Y=-P\left(\nabla_{Y} X\right)
$$

где $\nabla$ - риманова связность в римановом многообразии $M[58]$.

Лемма 3.4 .1 [50]. Пусть $\gamma: R \rightarrow M-$ геодезическая, лежащая в одном слое $T_{0}$, параметризованная длиной дуги, $X$ - касательное векторное поле $\kappa \gamma u \nabla^{\perp}$ - связность в ортогональном распределении $T_{0}^{\perp}$, индуцированная связностью $\nabla$. Тогда

$$
\nabla_{X}^{\perp}\left(C_{X}\right)=C_{X}^{2}+k(\operatorname{Id} \circ \gamma)
$$

где $k$ - константа.

ДокАЗАТЕЛЬСТво ЛЕммы 3.4.1. По определению $\nabla_{\gamma}^{\perp} Y=P\left(\nabla_{X} Y\right)$, где $Y \in T_{0}^{\perp}$, $X \in T M$. Для любого векторного поля $V$ и $U \in T_{0}$ верно

$$
P\left(\nabla_{U} V\right)=P\left(\nabla_{U}\left(V^{\prime}+P V\right)\right)=P\left(\nabla_{U} V^{\prime}\right)=P\left(\nabla_{U} P V\right),
$$

где $V=V^{\prime}+P V, V^{\prime} \in T_{0}$. Так как $T_{0}$ - геодезическое слоение, то $\nabla_{U} V^{\prime} \subset T_{0}$ и $P\left(\nabla_{U} V^{\prime}\right)=0$. Отсюда следует, что

$$
P\left(\nabla_{U} V\right)=P\left(\nabla_{U} P V\right) .
$$

Из формулы Вейнгартена следует, что $\nabla_{U} P V \subset T_{0}^{\perp}$ и

$$
P\left(\nabla_{U} V\right)=\nabla_{U} P V
$$

для любого $V \in T M, U \in T_{0}$.

Пусть $Y \subset T_{0}^{\perp}-$ произвольное векторное поле. Продолжим $X$ до векторного поля в $T_{0}$, определенного в открытой окрестности геодезической $\gamma$. По определению

$$
\left(\nabla_{X}^{\perp} C_{X}\right)(Y)=\nabla_{X}^{\perp}\left(C_{X}(Y)\right)-C_{X}\left(\nabla_{X}^{\perp} Y\right)=-\nabla_{X}^{\perp}\left(P\left(\nabla_{Y} X\right)\right)+P\left(\nabla_{\nabla_{X}^{\perp} Y} X\right)
$$

Ho

$$
\nabla_{X}^{\perp}\left(P\left(\nabla_{Y} X\right)\right)=P \nabla_{X} P\left(\nabla_{Y} X\right)
$$


Из (3.4.2) следует

$$
\nabla_{X}^{\perp}\left(P\left(\nabla_{Y} X\right)\right)=P\left(\nabla_{X} \nabla_{Y} X\right)
$$

И мы получим формулу

$$
\left(\nabla_{X}^{\perp} C_{X}\right)(Y)=-P\left(\nabla_{X} \nabla_{Y} X-\nabla_{\nabla_{X} Y} X\right) .
$$

Так как тензор кривизны $M$

$$
R(X, Y) Z=\nabla_{X} \nabla_{Y} Z-\nabla_{Y} \nabla_{X} Z-\nabla_{[X, Y]} Z,
$$

то

$$
R(X, Y) X=\nabla_{X} \nabla_{Y} X-\nabla_{Y} \nabla_{X} X-\nabla_{[X, Y]} X
$$

и

$$
\nabla_{X} \nabla_{Y} X=R(X, Y) X+\nabla_{Y} \nabla_{X} X+\nabla_{[X, Y]} X .
$$

Так как из условия 2) теоремы 3.4.2 следует, что $\langle R(X, Y) X, Y\rangle=-k$, то $P R(X, Y) X=-k Y$. Из этого условия и равенства

$$
[X, Y]=\nabla_{X} Y-\nabla_{Y} X
$$

следует, что

$$
\nabla_{X} \nabla_{Y} X=-k Y+\nabla_{Y} \nabla_{X} X+\nabla_{\nabla_{X} Y} X-\nabla_{\nabla_{Y} X} X .
$$

После подстановки в (3.4.5) мы получим

$$
\left(\nabla_{X}^{\perp} C_{X}\right)(Y)=k Y+P\left(\nabla_{Y} \nabla_{X} X-\nabla_{\nabla_{Y} X} X\right) .
$$

Пусть $Z$ есть векторное поле в $T_{0}^{\perp}$. Тогда

$$
Y\left\langle\nabla_{X} X, Z\right\rangle=\left\langle\nabla_{Y} \nabla_{X} X, Z\right\rangle+\left\langle\nabla_{X} X, \nabla_{Y} Z\right\rangle .
$$

Так как $\nabla_{X} X=0$, то

$$
\left\langle\nabla_{Y} \nabla_{X} X, Z\right\rangle=0 \text { и } P\left(\nabla_{Y} \nabla_{X} X\right)=0 .
$$

Мы получим

$$
\left(\nabla_{X}^{\perp} C_{X}\right)(Y)=P\left(\nabla_{\nabla_{Y} X} X\right)+k Y .
$$

По определению

$$
\nabla_{Y} X=P\left(\nabla_{Y} X\right)+X^{\prime}, \quad X^{\prime} \in T_{0},
$$

и

$$
P\left(\nabla_{P\left(\nabla_{Y} X\right)+X^{\prime}}\right)=P\left(\nabla_{P\left(\nabla_{Y} X\right)} X+\nabla_{X^{\prime}} X\right) .
$$

Но $\nabla_{X^{\prime}} X \subset L_{0}$ и $P\left(\nabla_{X^{\prime}} X\right)=0$.

Отсюда

$$
P\left(\nabla_{\nabla_{Y} X}\right)=P\left(\nabla_{P\left(\nabla_{Y} X\right)} X\right)=C_{X}^{2} .
$$

Мы получили, что

$$
\left(\nabla_{X}^{\perp} C_{X}\right)(Y)=C_{X}^{2}(Y)+k \operatorname{id}(Y),
$$

где id - тождественное отображение. 
ЛЕмма 3.4.2 [49]. Пусть $V$ - действительное $n$-мерное линейное пространство, $k$ - константа. Пусть $C:[a, b) \rightarrow \operatorname{end}(V)$ - дифференциальное отображение, удовлетворяющее уравнению

$$
C^{\prime}(t)=C^{2}(t)+k \text { id }
$$

для всех $t \in[a, b)$. Если $k>0$ и $n$-нечетно, то $b-a<\pi / \sqrt{k}$. Если существует собственное действительное значение оператора $C(t)$ при некотором значении $t_{0}$, то не существует регулярного решения матричного уравнения Риккати при всех значениях $t$ и $k>0$.

Лемма 3.4.3. Пусть $Q \in M^{n} u X \in T_{0}(Q)$. Ecли оператор $C_{X}$ имеeт coбcmвенное действительное значение, то $X=0$.

Эта лемма непосредственно следует из предыдущей леммы.

ДокаЗАТЕЛЬСТво ТеОРемЫ 3.4.2. Пусть $Q \in M$ и $X_{1}, \ldots, X_{\nu}$ - базис $T_{0}(Q)$. Определим операторы $C_{i}=C_{X_{i}}$, а также отображение $T_{0}^{\perp}(Q)-\{0\}$ в $\left(T_{0}^{\perp}(Q)\right)^{\nu+1}$ следуюшим образом:

$$
Y \rightarrow\left(Y, C_{1} Y, \ldots, C_{\nu} Y\right) .
$$

Эти $(\nu+1)$ векторов линейно независимы, поскольку в противном случае были бы справедливы равенства

$$
0=\alpha Y+\sum \alpha_{i} C_{i} Y=\alpha Y+C_{\sum \alpha_{i} X_{i}} Y
$$

и оператор $C$ имел бы действительное собственное значение с ненулевым собственным вектором. Это противоречит лемме 3.4.3.

Пусть $S^{n-\nu-1}$ - единичная сфера в $T_{0}^{\perp}$ в точке $Q$. Тогда векторные поля $C_{X_{i}} Y$ порождают линейно независимые векторные поля на сфере $S^{n-\nu-1}$. Их число $\rho(n-\nu)-1 \geqslant \nu ; \rho(n-\nu)>\nu$. Из определения $\nu(l)$ следует, что $\nu \leqslant \nu(l)$.

Теорему 3.4.2 можно переформулировать в эквивалентной форме как вопрос о структуре семейства линейных операторов.

Линейный оператор в четномерном пространстве $R^{n}$, как правило, не имеет собственных векторов. Ситуация меняется в случае $\nu$-параметрического семейства линейных операторов.

Лемма 3.4.4. Пусть $R^{\nu}, R^{n}(\nu, n>0)$ - вещественные евклидовьи пространства; B: $R^{\nu} \times R^{n} \rightarrow R^{n}-$ билинейный оператор. Тогда если линейные оператоpы $B(x, \cdot), x \neq 0$, не имеют вещ,ственных собственных значений, то $\nu \leqslant \rho(n)$.

В теореме 3.4.2 роль оператора $B$ играет конулевой оператор $C$.

Для евклидовых пространств с комплексной структурой справедлива

Лемма 3.4 .5 [33]. Пусть $J-$ комплексная структура на евклидовых пространствах $R^{\nu}, R^{n}(\nu, n>0)$ и $B: R^{\nu} \times R^{n} \rightarrow R^{n}-$ билинейньй оператор со свойством:

$$
B(J X, Y)=B(X, J Y)=J B(X, Y) .
$$

Тогда для любого $X \in R^{n}$ линейный оператор $B(X, \cdot)$ имеет вещественньй собственный вектор. 
ДокАЗАТЕльСТво ЛЕммы 3.4.5. Так как $B$ коммутирует с комплексной структурой $J$, то для любого ненулевого $X \in R^{n}$ найдется ненулевое комплексное число $\alpha+\beta j$ и ненулевой вектор $Y \in R^{n}$ со свойством $B(X, Y)=\alpha Y+\beta J Y$. Ввиду линейности $B$ для ненулевого вектора $Z=\alpha X-\beta J X$ вьполнено

$$
B(Z, Y)=\alpha B(X, Y)-\beta J B(X, Y)=\left(\alpha^{2}+\beta^{2}\right) Y,
$$

что и требовалось доказать.

Отсюда следует, что полные келеровы $k$-параболические подмногообразия в комплексном проективном пространстве устроены проще, чем в сферическом пространстве. Справедлива

ТЕОРема 3.4 .4 [33]. Пусть $F^{l}$ - полное келерово подмногообразие в комплексном проективном пространстве $\mathbb{C P}^{n}$. Если нуль-индекс $\mu(Q)>0$ во всех точках $F^{l}$, то подмногообразие $F^{l}$ является вполне геодезическим комплексным проективным пространством $\mathbb{C P}^{l / 2}$.

3.5. Сильно параболические подмногообразия в пространстве Лобачевского. В пространстве Лобачевского полных сильно параболических подмногообразий больше, чем в сферическом и евклидовом пространстве. Если мы возьмем интерпретацию Кэли-Клейна пространства Лобачевского в единичном шаре, то любой сильно параболической поверхности в евклидовом пространстве, особые и граничные точки которой лежат вне внутренности единичного шара, отвечает полная сильно параболическая поверхность пространства Лобачевского.

Изометрические погружения плоскости Лобачевского $L^{2}$ в пространство Лобачевского $L^{3}$ изучались в [57], пространства Лобачевского $L^{l}$ в $L^{l+1}-$ в [51]. Эти подмногообразия являются $(l-1)$-сильно параболическими. Если $\mu(Q)=l-1$, то кривая, ортогональная к полным вполне геодезическим слоям, имеет кривизну не больше 1 , где кривизна взята в метрике поверхности.

Более того, в [51] доказано: каждому вполне геодезическому слоению пространства Лобачевского $L^{l}$ на гиперповерхности отвечает изометрическое погружение $L^{l}$ в $L^{l+1}$, при котором слои переходят в слои нуль-распределения $(l-1)$-сильно параболической поверхности. В [57] были рассмотрены некоторые примеры полных сильно параболических поверхностей в пространстве Лобачевского $L^{3}$, которые были названы цилиндрическими поверхностями.

В многомерном пространстве Лобачевского есть подмногообразия, аналогичные цилиндрическим поверхностям в евклидовом пространстве. Мы найдем условия, когда сильно параболические поверхности в пространстве Лобачевского являются цилиндрами [15].

Пусть $H^{l+p-k}$ - либо орисфера, либо эквидистанта, либо вполне геодезическое пространство Лобачевского в пространстве $L^{l+p-k+1} ; F^{l-k}-$ полная регулярная поверхность в $H^{l+p-k}$. Проведем через точки $F^{l-k}$ полные геодезические в направлении нормалей к $H^{l+p-k}$ в $L^{l+p-k+1}$, получим поверхность $F^{l-k+1}$. Через точки поверхности $F^{l-k+1}$ проведем пространства Лобачевского $L^{k-1}$, перпендикулярные к $L^{l+p-k+1}$. Полученная полная поверхность $F^{l} \subset L^{l+p}$ является сильно $k$-параболической. Назовем ее цилиндром с $k$-мерной образующей. Цилиндрические вложения пространства Лобачевского $L^{2}$ в $L^{3}$ рассматривались в [57]. 
В интерпретации Кэли-Клейна, когда $F^{l-k}$ лежит на эквидистанте или в пространстве Лобачевского, цилиндрическая поверхность изображается цилиндром с $(k-1)$-мерной образуюшей над конической поверхностью $F^{l-k+1}$ с идеальной вершиной, лежащей вне абсолюта. Когда вершина лежит на бесконечности, будет цилиндр.

Если база $F^{l-k}$ расположена на орисфере, то поверхность $F^{l}$ изображается шилиндром с $(k-1)$-мерной образующей над конической поверхностью с вершиной на абсолюте. Образуюшие цилиндрической поверхности в евклидовом пространстве можно перевести друг в друга параллельным переносом. Аналогичньм свойством обладают цилиндрические поверхности в пространстве Лобачевского. Для этих поверхностей ортогональное к слоям сильной параболичности распределение $H(Q)$ голономно.

Интегральные многообразия $F^{l-k}$ являются вполне омбилическими на поверхности $F^{l}$, т.е. такими поверхностями, вторая квадратичная форма которых относительно произвольной нормали пропорциональна первой квадратичной форме [44].

Нормальная кривизна $k_{n}$ кривых, лежаших на многообразиях $F^{l-k}$, постоянна и в первом случае $0 \leqslant k_{n}<1$, и во втором, когда $F^{l-k}$ лежит на орисфере, $k_{n}=1$.

Метрика такой поверхности имеет вид

$$
d s^{2}=d s_{1}^{2}+\varphi^{2}\left(u^{1}, \ldots, u^{k}\right) d s_{2}^{2},
$$

где $d s_{1}^{2}$ - метрика пространства Лобачевского $L^{k}$, записанная в координатах $u^{1}, \ldots, u^{k} ; d s_{2}^{2}$ - метрика некоторой базы $F^{l-k}$; функция $\varphi$ в специальной системе координат совпадает с одной из следуюших функций:

$$
\operatorname{ch} \rho \operatorname{ch} u^{1}, \quad \operatorname{ch} \rho e^{-u_{1}}, \quad \operatorname{ch} \rho\left(\operatorname{ch} u_{1}+k(0) \operatorname{sh} u^{1}\right),
$$

где $\rho$ есть расстояние от фиксированной точки в $L^{k-1}$ и зависит только от координат $u^{2}, \ldots, u^{k}$.

Рассмотрим класс метрик, соответствуюших сильно параболическим поверхностям в пространстве постоянной кривизны.

Пусть $M^{n}$ - полное риманово многообразие. Внутренним нуль-индексом метрики $M^{n}$ называется число $\nu(Q)=\operatorname{dim} L(Q) \subset T_{Q} M^{n}$, где $L(Q)$ - максимальное подпространство касательного пространства такое, что

$$
R(X, Y) Z=c(\langle X, Z\rangle Y-\langle Y, Z\rangle X) \text { для любого } Y \in L(Q)
$$

где $X, Z \in T_{Q} M^{n}, R(X, Y)$ - оператор кривизны многообразия $M^{n}$. Локально эти метрики устроены следуюшим образом.

ЛЕмма 3.5.1 [55]. Пусть $M^{n}$ - риманово многообразие класса $C^{3} ; G$ - открьтая область, на которой внутренний нуль-индекс постоянен, $\nu(Q)=k$. Тогда распределение $L(Q)$ интегрируемо, слои являются областями на $k$-мерных вполне геодезических подмногообразиях постоянной кривизны с.

Если $M^{n}$ - полное многообразие и $\nu\left(Q_{0}\right)=\min _{Q \in F^{l}} \nu(Q)=k$, то слой $\mathscr{L}\left(Q_{0}\right)$ является полным вполне геодезическим подмногообразием. 
ЗАмЕчАниЕ. Мы приведем доказательство для случая $c=0$, хотя лемма справедлива для более общего случая, в частности, для нуль-пространств кривизноподобных тензоров. Тензор $K$ на римановом многообразии назьвается кривизноподобным, если удовлетворяет следуюшим условиям [55]:

1) $\left\langle K_{A B} X, Y\right\rangle=-\left\langle K_{A B} Y, X\right\rangle$;

2) $K_{A B}=-K_{B A}$;

3) $\left\langle K_{A B}(C), D\right\rangle=\left\langle K_{C D} A, B\right\rangle$;

4) $\sigma K_{A B} C=0$;

5) $\sigma\left(\nabla_{A} K\right)_{B C}=0$

где $X, Y, A, B, \ldots$ - векторные поля, $\sigma$ - циклическая перестановка. Свойства 1)-3) суть свойства симметрии и кососимметрии тензора кривизны, свойства 4), 5) суть первое и второе тождества Бианки. Если мы рассмотрим нуль-пространства кривизноподобного тензора и если они удовлетворяют условиям леммы, то распределение интегрируемо и слои являются вполне геодезическими подмногообразиями.

ДоКАЗАТЕЛЬСТВо ЛЕмМЫ 3.5.1. Пусть $X, Y$ - регулярные векторные поля на $M^{n}$ такие, что $X(Q), Y(Q) \subset L(Q)$. Выберем систему координат на $M^{n}$ так, чтобы в фиксированной точке $Q_{0}$ метрический тензор $g_{i j}=\delta_{i j}$ и символы Кристоффеля $\Gamma_{i j}^{k}\left(Q_{0}\right)=0$. Координаты векторных полей $X, Y$ удовлетворяют уравнениям:

$$
R_{i j k l} x^{k}=0 ; \quad R_{i j k l} y^{k}=0
$$

Продифференцируем (3.5.3) по координате $u^{s}$, где $\left(u^{1}, \ldots, u^{n}\right)$ - локальные координаты в окрестности точки $Q_{0}$; умножим на $y^{s}$ и просуммируем по $s$. Тогда в точке $Q_{0}$ мы получим

$$
\begin{aligned}
& R_{i j k l, s} x^{k} y^{s}+R_{i j k l} \frac{\partial x^{k}}{\partial u^{s}} y^{s}=0 \\
& R_{i j k l, s} y^{k} x^{s}+R_{i j k l} \frac{\partial y^{k}}{\partial u^{s}} x^{s}=0 .
\end{aligned}
$$

Вычтем (3.5.5) из (3.5.4). Тогда

$$
R_{i j k l}[X, Y]^{k}+R_{i j k l, s} x^{k} y^{s}-R_{i j s l, k} y^{s} x^{k}=0,
$$

где $[X, Y]$ - скобка Ли векторных полей $X, Y$,

$$
R_{i j k s} x^{k} y^{s}=0 \text {. }
$$

Отсюда следует, что

$$
R_{i j k s, l} x^{k} y^{s}=0 \text {. }
$$

Из второго тождества Бианки следует, что

$$
R_{i j k l, s}+R_{i j s k, l}+R_{i j l s, k}=0 .
$$


Из (3.5.8), (3.5.9) следует, что

$$
R_{i j k l, s} x^{k} y^{s}-R_{i j s l, k} x^{k} y^{s}=-R_{i j s k, l} x^{k} y^{s}=0
$$

Поэтому из (3.5.6) следует, что в точке $Q_{0}$

$$
R_{i j k l}[X, Y]^{k}=0 \text { и }[X, Y] \subset L\left(Q_{0}\right) .
$$

В силу произвольности выбора точки $Q_{0}$ это условие вьполняется в открытой окрестности. Из теоремы Фробениуса следует, что распределение $L(Q)$ интегрируемо. Выберем координаты в римановом многообразии так, чтобы слои были координатными поверхностями $u^{1}, \ldots, u^{k}$.

Тогда

$$
R_{i j \alpha m}=0 ; \quad \alpha=1, \ldots, k ; \quad i, j, m, s=1, \ldots, n .
$$

Отсюда следует, что

$$
R_{i j \alpha m, s}=0 .
$$

Taк как

$$
R_{i j \alpha l} x^{\alpha}=0
$$

TO

$$
R_{i j \alpha l} \nabla_{s} x^{\alpha} y^{s}=0
$$

То есть

$$
R_{i j \alpha l}\left(\nabla_{y} X\right)^{\alpha}=0
$$

Отсюда следует, что

$$
\nabla_{Y} X \subset L(Q)
$$

и слоение является вполне геодезическим.

Пусть многообразие $M^{n}$ - полное, и пусть $U$ - открытое множество, где внутренний нуль-индекс $\nu$ принимает минимальное значение.

Пусть $\gamma$ - геодезическая линия, принадлежашая слою $L(Q)$. Докажем, что слой будет полным. В силу полноты многообразия $M^{n}$ геодезическую $\gamma$ можно неограниченно продолжить. Если слой $L(Q)$ не полньй, то на геодезической $\gamma$ найдется граничная точка $Q_{0}$. Выберем в окрестности геодезической линии координаты Ферми, чтобы на геодезической $g_{i j}=\delta_{i j}, \Gamma_{i j}^{k}=0$. Вдоль геодезической параметром является $u^{1}$ - длина дуги. Пусть $X_{0} \subset L\left(Q_{0}\right)$ - вектор в точке $Q_{0}$, принадлежащий нуль-пространству. Параллельно перенесем этот вектор вдоль $\gamma$ в точку $Q$ и получим векторное поле $X$,

$$
\begin{gathered}
\nabla_{1}\left(R_{i j k l} x^{s}\right)=R_{i j k l, 1} x^{s}, \\
R_{i j k l, 1}+R_{i j 1 k, l}+R_{i j l 1, k}=0, \\
R_{i j 1 k, l}=R_{i j l 1, k}=0 .
\end{gathered}
$$

Отсюда следует, что

$$
\nabla_{1}\left(R_{i j k l} x^{s}\right)=0
$$


То есть вектор $X$ в точке $Q$ принадлежит нуль-пространству. Отсюда следует, что

$$
\nu\left(Q_{0}\right) \leqslant \nu(Q) .
$$

В силу минимальности нуль-индекса в точке $Q$ нуль-индекс $\nu\left(Q_{0}\right)=k$ и является также минимальным. Тогда в окрестности точки $Q_{0}$ нуль-индекс постоянный и точка $Q_{0}$ является внутренней точкой слоя $L\left(Q_{0}\right)$, которьй совпадает со слоем $L(Q)$. Отсюда следует, что геодезическую $\gamma$ можно продолжить за точку $Q_{0}$ и слой является полным римановым многообразием.

Справедлива

Теорема 3.5.1. Пусть $M^{l}$ - полное риманово многообразие с постоянныцм внутренним нуль-индексом $\nu=k>0$.

Если существует поверхность, ортогональная слоям вполне геодезичности $L^{k}(Q), u$

1) $c>0$, то $\nu=l$ и $M^{l}$ локально изометрична сфере радиуса $1 / \sqrt{l}$; при условии односвязности есть глобальная изометрия;

2) $c=0$, то $M^{l}$ есть метрическое произведение риманова многообразия $M^{l-k}$ и евклидова пространства $E^{k}$

3) $c<0$ (не ограничивая общности, можнно считать $c=-1)$ и поверхность, ортогональная слою, вполне омбилическая при $k<l-1 ;$ при $k=l-1$ кривая, ортогональная слою, имеет постоянную кривизну, то $M^{l}$ полупроводимо и его метрика имеет вид (3.5.1), где $\varphi$ - указанные выше функиии.

В доказательстве теоремы используется полнота риманова многообразия в окрестности одного слоя и доказьвается строение метрики в окрестности этого слоя.

Имеются аналогичные утверждения для поверхностей в пространствах постоянной кривизны.

ТеОРема 3.5.2. Пусть $F^{l}$ - полная регулярная класса $C^{3}$ сильно параболическая поверхность с постоянныц внешним нуль-индексом $\mu=k$ в односвязном пространстве постоянной кривизны $R^{n}(c)$. Если существует поверхность, ортогональная слоям вполне геодезичности $L^{k}(Q), u$

1) $c>0$, то $\mu=l$ и $F^{l}$ - вполне геодезическая большая сфера в сферическом пространстве;

2) $c=0$, то $F^{l}$ есть иилиндр с k-мерной образующей в евклидовом пространстве;

3) $c<0$ (не ограничивая общности, можно считать $c=-1$ ) и поверхность, ортогональная слою, вполне омбилическая как подмногообразие $F^{l}$ при $k<l-1 ;$ при $k=l-1$ кривая, ортогональная слою, имеет постоянную геодезическую кривизну, то $F^{l}$ есть иилиндрическая поверхность в пространстве Лобачевского, которая в интерпретации Кәли-Клейна изображсается иилиндром $с(k-1)$-мерной образующей над конической поверхностью с вершиной либо на абсолюте, либо вне его. 
ДоКАЗАТЕЛЬСТВо ТЕОРЕМЫ 3.5.1. Пусть $X(Q)=k$ и ортогональное распределение $H(Q)$ голономно. Тогда по лемме 3.5.1 метрика $M^{n}$ имеет вид

$$
\begin{gathered}
d s^{2}=d s_{1}^{2}+d s_{2}^{2}=g_{i j} d u^{i} d u^{j}+g_{\alpha \beta} d v^{\alpha} d v^{\beta}, \\
i, j=1, \ldots, k ; \quad \alpha, \beta=k=1, \ldots, l,
\end{gathered}
$$

где $d s_{1}^{2}$ - метрическая форма вполне геодезического слоя $L^{k}(Q)$, изометричного пространству постоянной кривизны $R^{k}(c)$. Так как вторые квадратичные формы слоя $R^{k}(Q)$ относительно ортонормированных нормалей имеют вид

$$
A_{i j}^{\alpha}=-\frac{1}{2} \frac{\partial g_{i j}}{\partial v^{\alpha}}=0
$$

то $g_{i j}$ зависят только от $u^{1}, \ldots, u^{k}$ и в некоторой системе координат имеют стандартные значения.

В координатной записи условия (3.5.2) имеют следующий вид:

$$
\begin{aligned}
& R_{\alpha \nu i j}=0 \\
& R_{i \alpha j \beta}=c g_{i j} g_{\alpha \beta} \\
& R_{i \alpha \beta \nu}=0
\end{aligned}
$$

где $R_{A B C D}, A, B, C, D=1, \ldots, l,-$ компоненты тензора кривизны многообразия $M^{n}$. Явно вычислив $R_{\alpha \beta i j}$ через компоненты метрического тензора $g_{A B}$ и учитьвая равенство $g_{\alpha j}=0$ и соотношения (3.5.10), получим

$$
\frac{\partial g_{\alpha \nu}}{\partial u_{j}} \frac{\partial g_{\beta \delta}}{\partial u_{i}}-\frac{\partial g_{\beta \nu}}{\partial u_{j}} \frac{\partial g_{\alpha \delta}}{\partial u_{i}}=0
$$

Коэффициенты вторых квадратичных форм поверхностей $u^{i}=$ const $(i=1, \ldots, k)$ относительно ортонормированного базиса единичных нормалей, направленных по координатным осям $u^{i}$, имеют вид [31, с. 191]

$$
A_{\alpha \beta}^{i}=-\frac{1}{2} \frac{\partial g_{\alpha \gamma}}{\partial u^{j}}
$$

Запишем уравнения Риччи для ортогональных слоев $F^{l-k}$ :

$$
K_{\alpha \beta i j}=R_{\alpha \beta i j}-g^{\alpha \beta}\left(A_{\alpha \gamma}^{i} A_{\beta \delta}^{j}-A_{\alpha \delta}^{i} A_{\beta \gamma}^{j}\right),
$$

где $K_{\alpha \beta i j}$ - тензор кривизны нормальной связности поверхности $F^{l-k}$. Из (3.5.11), (3.5.14), (3.5.15) следует, что слои $F^{l-k}$ имеют нормальную связность.

Пусть у нас будет лишь один слой $F^{l-k}$, ортогональньй слоям сильной параболичности $L^{k}(Q)$. Из тех же рассуждений следует, что $F^{l-k}$ будет иметь плоскую нормальную связность. Пусть $N^{i}$ - параллельнье в нормальной связности ортонормированные векторные поля вдоль $F^{l-k}$. Вдоль них в каждом вполне геодезическом слое будут вьпущены координатные геодезические линии, координаты $u^{i}$ являются нормальными координатами Ферми в каждом слое, т.е. координаты $u^{i}$ точки слоя - 
это декартовы координаты точки касательного пространства к слою $L(Q)$ с началом координат в точке $Q \in F^{l-k}$ с ортогональньм базисом $N^{i}$, которая при экспоненциальном отображении переходит в данную точку слоя; $v^{\alpha}-$ координаты на поверхности $F^{l-k} ;\left(u^{i}, v^{\alpha}\right)-$ координаты в римановом многообразии $M^{n}$. Докажем, что слои $u^{i}=$ const $(i=1, \ldots, k)$ ортогональны слоям $L^{k}(Q)$. Рассмотрим семейство геодезических линий, выходящих в направлении векторного поля $N^{i}$, геодезическая $\gamma=\gamma(t)$ - фиксированная кривая из этого семейства, $Y(t)$ - поле Якоби вдоль геодезической $\gamma$, порожденное данньм семейством. Так как $\gamma(t) \subset L(Q)$, то вдоль этой геодезической

$$
R(\dot{\gamma}, Y) \dot{\gamma}=c[\langle\dot{\gamma}, \dot{\gamma}\rangle Y-\langle\dot{\gamma}, Y\rangle \dot{\gamma}]
$$

Поэтому уравнение $Y^{\prime \prime}+R(\dot{\gamma}, Y) \dot{\gamma}=0$, которому удовлетворяет $Y(t)$, совпадает с уравнением для полей Якоби в пространстве постоянной кривизны. Значит, поле Якоби $Y(t)$ в каждой точке $\gamma(t)$ лежит в плоскости, натянутой на векторы, полученные из векторов $Y(0), \nabla_{\dot{\gamma}(Q)} Y(t)$ параллельным переносом вдоль геодезической $\gamma[21]$.

Так как $Y(t)$ касается слоя $F^{l-k}$, то $Y(0)$ ортогонален геодезической. Поле $Y(t)$ удовлетворяет следуюшему уравнению:

$$
\nabla_{\dot{\gamma}(0)} Y(t)-\nabla_{Y(0)} N^{i}-\left[Y(t), N^{i}\right]=0, \quad\left[Y(t), N^{i}\right]=0,
$$

так как $Y(t), N^{i}$ - векторы, касательные координатным линиям, вектор $\nabla_{Y(0)} N^{i}$-касательньй к слою, поскольку векторное поле $N^{i}$ - параллельное в нормальной связности. Поэтому вектор $\nabla_{\dot{\gamma}(Q)} Y(t)$ перпендикулярен к геодезической $\gamma(t)$. Отсюда следует, что поле Якоби $Y(t)$ перпендикулярно к геодезической при любом значении параметра. С другой стороны, $Y(t)$ является касательным векторным полем к слоям $u^{i}=$ const $(i=1, \ldots, k)$. Поэтому в указанной системе координат $g_{i \alpha}=0$. Таким образом, необходимьм и достаточным условием голономности ортогонального распределения $H(Q)$ является наличие хотя бы одного слоя, ортогонального вполне геодезичньп слоям.

Уравнения (3.5.12), (3.5.13) в терминах второй квадратичной формы $F^{l-k}$ переписьваются следующим образом:

$$
\begin{gathered}
\frac{\partial A_{\alpha \beta}^{i}}{\partial u^{j}}=g^{\gamma \delta} A_{\beta \delta}^{i} A_{\alpha \gamma}^{j}+c g_{i j} g_{\alpha \beta} ; \\
A_{\alpha \beta, \gamma}^{i}-A_{\alpha \gamma, \beta}^{i}=0,
\end{gathered}
$$

где ковариантные производные берутся в метрике слоя. Выберем координаты в $M^{n}$ так, чтобы вдоль геодезической, направленной по координатной линии $u^{i}$, вьполнялись равенства $g_{i j}=\delta_{i j}, g_{\alpha \beta}=\delta_{\alpha \beta}$. Тогда уравнения (3.5.17) при $i=j, \alpha=\beta$ имеют вид

$$
\frac{\partial A_{\alpha \alpha}^{i}}{\partial u^{i}}=\left(A_{\alpha \alpha}^{i}\right)^{2}+c .
$$

Это есть уравнение Риккати, которое в силу полноты риманова многообразия $M^{n}$ должно иметь регулярное решение на всей оси $u^{i}$. При $c=0$ единственное такое решение $A_{\alpha \alpha}^{i}=0$. В силу произвольности выбора $i, \alpha$ и точки слоя $F^{l-k}$ вторая квадратичная форма слоя тождественно равна нулю, и любой слой является вполне геодезическим подмногообразием. Отсюда следует, что $M^{n}$ - метрическое произведение 
$F^{l-k}$ на евклидово пространство. При $c>0$ регулярных решений нет и $M^{n}$ локально изометрично сфере радиуса $1 / \sqrt{c}$, при условии односвязности $M^{n}$ оно будет глобально изометрично сфере.

При $c<0, c=-k_{0}^{2}$ решение $k=A_{\alpha \alpha}^{i}$ имеет вид

$$
k=k_{0} \frac{\left(k(0)+k_{0}\right)+\left(k(0)-k_{0}\right) e^{2 k_{0} u^{1}}}{\left(k(0)+k_{0}\right)-\left(k(0)-k_{0}\right) e^{2 k_{0} u^{1}}} .
$$

Оно будет всюду регулярньп, если $k(0) \leqslant k_{0}$.

При $c=-1$ будет $k(0) \leqslant 1$. Геометрически это значит, что нормальная кривизна любой кривой слоя, ортогонального вполне геодезичным слоям, менњше либо равна единице, где под нормальной кривизной понимается модуль вектора $A(x, x)$ с компонентами $A_{\alpha \beta}^{i} x^{\alpha} x^{\beta}$, где $X$ - единичньй касательньй к поверхности $F^{l-k}$ вектор с компонентами $x^{\alpha}$. Если для некоторого слоя при $c=-1$ нормальная кривизна меньше единицы, то она будет меньше единицы для всех слоев и будет стремиться к единице при уходе на бесконечность. Если для некоторого слоя она равна единице, то это справедливо для каждого слоя.

Рассмотрим случай $c=-1$. Пусть фиксированная поверхность $F^{l-k}$ вполне омбилична. Тогда в каждой точке существует единственная нормаль $n_{1}$, относительно которой вторая квадратичная форма нетривиальна, а относительно перпендикулярных ей нормалей $n_{2}, \ldots, n_{k}$ вторые квадратичные формы равны нулю. Пусть $n_{i}-$ такие нормали в фиксированной точке $P_{0} \in F^{l-k}$. Параллельно перенесем в нормальной связности нормали $n_{i}$ вдоль поверхности $F^{l-k}$, получим нормальные поля $N_{i}$. В точ$\operatorname{Kax} F^{l-k}$

$$
A_{\alpha \beta}^{1+i}=\mu^{i} A_{\alpha \beta}^{1} ; \quad A_{\alpha \beta}^{1}(0)=\lambda g_{\alpha \beta} ; \quad A_{\alpha \beta}^{1+i}(0)=0 .
$$

Из (3.5.18) и (3.5.21) следует, что

$$
\frac{\partial \lambda}{\partial v^{\gamma}} g_{\alpha \gamma}-\frac{\partial \lambda}{\partial v^{\beta}} g_{\alpha \gamma}=0 ; \quad \frac{\partial \mu^{i}}{\partial v^{\gamma}} g_{\alpha \beta}-\frac{\partial \mu^{i}}{\partial v^{\beta}} g_{\alpha \gamma}=0
$$

Выбрав в произвольной точке $P \in F^{l-k}$ координаты так, чтобы $g_{\alpha \beta}=\delta_{\alpha \beta}, \alpha=$ $\beta \neq \gamma$, из (3.5.22) получим

$$
\frac{\partial \lambda}{\partial v^{\gamma}}=0 ; \quad \frac{\partial \mu^{i}}{\partial v^{\gamma}}=0
$$

Поэтому, с учетом (3.5.22), получим

$$
\lambda=\text { const } ; \quad \mu^{i}=0 .
$$

Направим координатные линии $u^{i}$ вдоль векторных полей $N_{i}$, причем параметр $u^{1}-$ длина геодезической, выходящей в направлении вектора $N_{1}$.

Так как

$$
\frac{\partial A_{\alpha \beta}^{i}}{\partial u_{i}}=-\frac{1}{2} \frac{\partial g_{\alpha \beta}}{\partial u^{i}},
$$


то из (3.5.24) получим

$$
\frac{\partial g_{\alpha \beta}}{\partial u^{2}}=\frac{\partial g_{\alpha \beta}}{\partial u^{k}}=0 ; \quad \frac{\partial g_{\alpha \beta}}{\partial u^{1}}=-2 \lambda g_{\alpha \beta}
$$

С другой стороны, вторые квадратичные формы базы цилиндрической поверхности в пространстве Лобачевского имеют такой же вид, как вторые квадратичные формы омбилической поверхности. Метрический тензор риманова многообразия $M^{n}$ удовлетворяет тем же дифференциальным уравнениям, что и метрический тензор цилиндрической поверхности, с теми же начальными условиями. Отсюда следует, что их метрики совпадают.

Если ортогональньй слой одномерен, то для получения тех же выводов необходимо потребовать постоянства его кривизны в $M^{n}$.

Нормальные векторные поля $N^{1+i}$ вдоль ортогонального слоя параллельны в нормальной связности, и из (3.5.24) следует, что вторые квадратичные формы относительно них равны нулю. Поэтому из формул Вейнгартена

$$
\nabla_{\beta} N^{i}=-g^{\alpha v} A_{\beta \alpha}^{i} r_{\gamma}+\nabla_{\beta}^{\perp} N^{i}=0
$$

где $\nabla_{\beta}$ - ковариантная производная в римановом пространстве в координатном направлении слоя; $\nabla^{\perp}-$ ковариантная производная нормальной связности. Значит, они параллельны в связности многообразия $M^{n}$. Координатная поверхность переменных $u^{1}, v^{k+1}, \ldots, v^{l}$ является вполне геодезической поверхностью $M^{n}$.

Доказательство теоремы 3.5.2 проходит аналогично доказательству теоремы 3.5.1. Поэтому

1) $\mu=l$ и $F^{l}$ является большой сферой в сферическом пространстве при $c>0$;

2) при $c=0$ из теоремы 3.5.1 следует, что $F^{l}$ есть метрическое произведение $F^{l-k}$ на евклидово пространство $E^{k}$. Но так как евклидово пространство $E^{k}$ реализовано плоскостью объемлющего евклидова пространства $E^{n}$, то $F^{l}$ есть $k$-мерньй цилиндр [7];

3) при $c=-1$ построенные в теореме 3.5.1 векторные поля $N^{1+i}$ будут параллельны в объемлюшем пространстве Лобачевского.

По формуле Вейнгартена $\widetilde{\nabla}_{\alpha} N^{1+i}=\nabla_{\alpha} N^{i}+A\left(e_{\alpha}, N^{i}\right)=0$, где $\widetilde{\nabla}_{\alpha}-$ ковариантная производная в направлении ортогонального слоя в пространстве Лобачевского, $e_{\alpha}$ - вектор, касательный к координатной линии $v^{\alpha}$. Первое слагаемое равно нулю в силу (3.5.26), второе - так как $N^{i} \subset L(Q)$ из-за сильной параболичности поверхности. Поэтому поверхность $F^{l-k}$ лежит в пространстве Лобачевского $L^{l+p-k+1}$. Нормальное векторное поле $N^{1}$, относительно которого вторая квадратичная форма $F^{l-k}$ омбилична, параллельно в нормальном расслоении $F^{l-k} \subset L^{l+p-k+1}$.

В силу уравнений Кодащци нормальная кривизна поверхности в каждой точке относительно любого направления постоянна и удовлетворяет неравенству $0 \leqslant k_{n} \leqslant 1$. Пусть $n_{q}$ - ортонормированные нормали к поверхности $F^{l-k}$ в $L^{l+p-k+1}$, ортогональные $N^{1}, A_{\alpha \beta}^{q}$ - коэффициенты вторых квадратичных форм относительно этих нормалей. Метрика, вторые квадратичные формы $A_{\alpha \beta}^{q}$, коэффициенты кручения $\mu_{q r, \alpha}$ поверхности $F^{l-k}$ в силу свойств нормального векторного поля $N^{1}$ удовлетворяют 
уравнениям Гаусса-Петерсона-Кодацши-Риччи в пространстве постоянной кривизны. Поэтому существует реализация метрики $F^{l-k}$ поверхностью $\bar{F}^{l-k}$ в $H^{l+p-k} \subset$ $L^{l+p-k+1}$, где $H^{l+p-k}$ при $k_{n}=0$ есть пространство Лобачевского $L^{l+p-k}$, при $0<k_{n}<1$ - эквидистанта, при $k_{n}=1$ - орисфера.

Если рассматривать $\bar{F}^{l-k}$ как поверхность в $L^{l+p-k+1}$, то все квадратичные формы и коэффициенты кручения поверхностей $F^{l-k}$ и $\bar{F}^{l-k}$ совпадут. Поэтому поверхности совпадают с точностью до движения. Через каждую точку $F^{l-k}$ проведены геодезические в направлении нормалей к $H^{l+p-k}$. Они образуют вполне геодезическую поверхность $F^{l-k+1} \subset L^{l+p-k+1} ; F^{l-k+1} \subset F^{l}$. Через каждую точку $F^{l-k+1}$ проходит пространство Лобачевского $L^{k-1}$, ортогональное $L^{l+p-k+1}$, натянутое на параллельные поля $N^{i}$. Отсюда и следует, что $F^{l}$ является цилиндром в пространстве Лобачевского.

3.6. Сильно параболические подмногообразия в псевдоевклидовом и псевдоримановом пространствах постоянной кривизны. Эти подмногообразия изучались рядом авторов. Л. Грейвс обобшил теорему Хартмана-Ниренберга на случай изометрического погружения псевдоевклидова пространства $E_{1}^{n}$ с сигнатурой $(-,+, \ldots,+)$ в псевдоевклидово пространство $E_{1}^{n-1}$ с этой же сигнатурой. В этом случае нуль-индекс $\mu \geqslant n-1$ [53].

Для сильно параболических подмногообразий с ограничением на кривизну Риччи справедлива

Теорема 3.6.1 [37]. Пусть $M_{s}^{l}$ есть геодезически полное подмногообразие сигнатуры $(s, l-s)$ в псевдоевклидово пространство $E_{s}^{n}$ сигнатуры $(s, n-s)$. Предположим, что нуль-индекс $\mu(Q)=k$ постоянен на подмногообразии $M_{s}^{l}$ и кривизна Риччи $\operatorname{Ric}(X, X) \geqslant 0$ для всех времениподобных векторов.

Если существует точка $Q_{0}$, для которой нуль-пространство $L^{k}\left(Q_{0}\right)$ есть псевдоевклидова плоскость $E_{s}^{k}$, то подмногообразие $M_{s}^{l}$ есть иилиндр с $k$-мерной образующей $E_{s}^{k}$.

ЗАмечАнИЕ. Интересно выяснить строение подмногообразий, когда нуль-пространство евклидово или имеет вырожденную метрику.

ДоКАЗАТЕЛЬСТВо ТЕОРЕМЫ 3.6.1. Аналогично риманову случаю для псевдоримановых пространств постоянной кривизны доказано, что нуль-распределение интегрируемо, слои являются вполне геодезическими подмногообразиями и при вьполнении условий теоремы геодезически полными [35].

Пусть $x_{1}, \ldots, x_{s}, x_{s+1}, \ldots, x_{n}$ - ортогональная декартова система координат в $E_{s}^{n}$. Рассмотрим наложенное евклидово пространство $E^{n}$ с этой же ортогональной декартовой системой координат. Пусть $\bar{M}^{l}$ - соответствующее подмногообразие в $E^{n}$. Из [11] следует, что вторые квадратичные формы $M_{s}^{l}$ и $\bar{M}^{l}$ пропорциональны, нуль-индекс $\bar{M}^{l} \quad \mu=k$ и $\bar{M}^{l}$ полно вдоль вполне геодезических слоев. Пусть $Q_{0} \in M_{s}^{l}$ есть точка, в которой нуль-пространство $L^{k}\left(Q_{0}\right)$ есть псевдоевклидова плоскость $E_{s}^{k}$. Тогда в окрестности соответствуюшего слоя $E^{k}\left(Q_{0}\right)$ на $M^{l}$ по условию теоремы кривизна Риччи неотрицательна. Из леммы 3.3 .1 следует, что $j(Q)=0$. Из пропорциональности вторых квадратичных форм и из теоремы 3.3 .1 следует, что $\bar{M}^{l}$ в окрестности 
слоя $E^{k}\left(Q_{0}\right)$ есть цилиндр с образующей $E^{k}$. Из постоянства нуль-индекса и условия на кривизну Риччи следует, что $\bar{M}^{l}$ в целом есть цилиндр. Это верно для соответствующего подмногообразия $M_{s}^{l}$, образующей цилиндра будет псевдоевклидова плоскость $E_{s}^{k}$.

С. Яу была высказана гипотеза о том, что теорема Чигера-Громолла верна для геодезически полных лоренцевых 4-мерных многообразий неотрищательной кривизны Риччи во времениподобных направлениях, которые содержат максимальную времениподобную геодезическую.

Первые результаты в этом направлении получены Ешенбургом и Галловеем. В 1990 году Ньюмен получил окончательный результат [56].

Пусть $\left(M^{n}, g\right)$ есть времениподобное геодезически полное псевдориманово многообразие с сигнатурой $(-,+, \ldots,+)$. Предположим, что $\operatorname{Ric}(X, X) \geqslant 0$ для всех времениподобных векторов и $M^{n}$ содержит времениподобную максимальную геодезическую $\alpha: R \rightarrow M^{n}$.

Тогда $\left(M^{n}, g\right)$ изометрично $\left(R \times \widetilde{M}^{n-1},-d t^{2} \oplus h\right)$, где $\left(\widetilde{M}^{n}, h\right)$ есть полное риманово многообразие и $\alpha$ задано в виде $t \rightarrow(t, p)$ для некоторой точки $p \in \widetilde{M}$.

Имеет место внешний аналог этой теоремы аналогично евклидову случаю (лемма 3.3.2). Справедлива

Теорема 3.6.2 [37]. Пусть $M_{1}^{l}$ есть времениподобное геодезически полное подмногообразие псевдоевклидова пространства $E_{1}^{n}$. Предположим, что

1) $\operatorname{Ric}(X, X) \geqslant 0$ для всех времениподобных векторов;

2) подмногообразие $M_{1}^{l}$ содержит времениподобную прямую линию обгемлющего пространства $E_{1}^{n}$.

Тогда подмногообразие $M_{1}^{l}$ есть иилиндр, который расщепляется как метрическое произведение $\left(E_{1}^{1} \times \widetilde{M}^{n-1},-d t^{2} \oplus h\right)$, где $\widetilde{M}^{l-1} \subset E^{n-1}, h-$ индуцированная метрика на $\widetilde{M}$ и $E_{1}^{1}-$ времениподобная прямая.

Аналогично лемме 3.3.3 для евклидова случая имеет место

ЛЕмма 3.6.1 [37]. Предположим, что $M_{1}^{2}$ есть поверхностькласса $C^{1}$ в псевдоевклидовом пространстве $E_{1}^{n}$, изометричная полосе на лоренцевой плоскости $E_{1}^{2}$ с сигнатурой $(+,-)$. Если существует времениподобная прямая обгемлющего пространства, которая лехит на поверхности $M_{1}^{2}$, то поверхность есть иилиндр с времениподобной образующей.

ДоКАЗАТЕЛЬСТВо тЕОРемы 3.6.2. Из результата Ньюмена следует, что $M_{1}^{l}$ изометрично $\left(R \times \widetilde{M}^{l-1},-d t^{2} \oplus h\right)$, где $\left(\widetilde{M}^{l-1}, h\right)$ есть полное риманово многообразие и $\alpha: R \rightarrow M^{l}$ задается в виде $\alpha(t)=(t, p)$ для некоторой $p \in \widetilde{M}^{l-1}$. Пусть $\gamma-$ некоторая кривая на $\widetilde{M}^{l-1}$. Тогда $R \times \gamma$ изометрично полосе на лоренцевой плоскости. Из леммы 3.6.1 следует, что $M_{1}^{l}$ есть цилиндр в псевдоевклидовом пространстве $E_{1}^{n}$.

Когда объемлющим пространством является псевдориманово пространство $M_{s}^{n}$ постоянной положительной кривизны, доказаны аналоги соответствуюших теорем 3.4.1, 3.4.2 в сфрерическом пространстве. Однако отличие заключается в том, что для сильно параболических подмногообразий в псевдоримановом пространстве $M_{1}^{n}$ нуль-пространство может быть

1) псевдоевклидовой плоскостью $E_{1}^{k}$, 
2) евклидовой плоскостью $E^{k}$,

3) плоскостью с вырожденной метрикой.

От этого существенно зависит окончательньй результат.

ТЕОРема 3.6 .3 [35]. Пусть $M_{1}^{n}$ - геодезически полное лоренцево подмногообразие в лоренцевом подмногообразии $\widetilde{M}_{1}^{n+p}(c)$ постоянной положительной кривизньи, пусть

$$
\mu_{0}=\min _{Q \in M_{1}^{l}} \mu(Q)
$$

Предположим, что кривизна Риччи $M_{1}^{l}$

$$
\operatorname{Ric}(X, X) \geqslant(l-1) c
$$

для всех единичных пространственноподобных векторов.

1) Если в некоторой точке $Q \in M_{1}^{l}\left(\mu(Q)=\mu_{0}\right)$ нуль-пространство $T^{0}(Q)$ лоренцево, то $\mu_{0}-$ или 0 , или $l$.

2) Если в некоторой точке $Q \in M_{1}^{l}\left(\mu(Q)=\mu_{0}\right)$ нуль-пространство вырождено, то нуль-индекс $\mu_{0}$ может принимать только лишь следующие значения: $0,1, n-1$ или $n$.

3) Если в некоторой точке $Q \in M_{1}^{l}\left(\mu(Q)=\mu_{0}\right)$ нуль-пространство евклидово и $p=1$, то нуль-индекс $\mu_{0}$ может принимать только лишь значения: $0, n-2$, $n-1$ или $n$.

При доказательстве этой теоремы также вводится конулевой оператор $C_{X}$, которьй удовлетворяет уравнению Риккати. В предположениях на $\mu_{0}$, не удовлетворяющих условиям теоремы, оператор $C_{X}$ имеет действительное собственное значение, при этом $X$ может быть выбран пространственноподобньм [35]. Но уравнение

$$
\frac{d C(t)}{d t}=C^{2}(t)+c \cdot \mathrm{id}
$$

не имеет глобального решения, когда $c>0$ и $C_{X}$ имеет действительное собственное значение. С другой стороны, слой является вполне геодезическим полньм подмногообразием и вдоль пространственноподобной геодезической уравнение Риккати имеет глобальное регулярное решение. Отсюда следует невозможность других значений $\mu_{0}$, кроме приведенных в условиях теоремы.

Без ограничений на кривизну подмногообразия справедлив аналог теоремы 3.4.2, доказанной Ферусом.

Определим $\nu_{n}-$ наибольшее число, удовлетворяюшее неравенству

$$
\rho\left(n-\bar{\nu}_{n}\right) \geqslant \bar{\nu}_{n}
$$

где $\rho(l)-1$ есть число линейно независимых векторных полей на сфере $S^{l-1}$.

Раньше мы определили число $\nu_{n}$ как наибольшее число, удовлетворяющее неравенству

$$
\rho\left(n-\nu_{n}\right)-1 \geqslant \nu_{n} .
$$

Ясно, что $\nu_{n} \leqslant \bar{\nu}_{n}$. 
ТЕОРема 3.6.4 [35]. Пусть $F_{1}^{l}$ есть геодезически полное связное подмногообразие $\widetilde{M}_{1}^{n+p}(c), c>0$. Если нуль-индекс $\mu_{0}>\bar{\nu}(l)$, то $F_{1}^{l}$ есть вполне геодезическое подмногообразие в $\widetilde{M}_{1}^{n+p}(c) u \mu_{0}=l$.

Доказательство полностью аналогично сферическому случаю.

Этот результат обобшается также на келеровы подмногообразия в псевдоримановом комплексном проективном пространстве $\mathbb{C P}_{k}^{n}[36]$.

ТеОРема 3.6.5. Пусть $F^{l}$ - комплексное l-мерное келерово подмногообразие в $\mathbb{C P}_{k}^{n}$. Предположим, что $F^{l}$ или геодезически полное, или замкнутое в $\mathbb{C P}_{k}^{n}$.

Если нуль-индекс

$$
\mu_{0}=\min _{Q \in F^{l}} \mu(Q)
$$

больше, чем сигнатура метрики $\mathbb{C P}_{k}^{n}, \mu_{0}>k$, mо $F^{l}=\mathbb{C P}_{t}^{l}$ для некоторого $0 \leqslant t \leqslant k$ и $F^{l}-$ вполне геодезическое подмногообразие.

ДоКАЗАТЕЛЬСТВо ТЕОРЕМЫ 3.6.5. Предположим, что $l>\mu_{0}>k$. Тогда через точку $Q_{0}$, где $\mu\left(Q_{0}\right)=\mu_{0}$, проходит вполне геодезическое пространство $\mathbb{C P}_{s}^{\mu_{0}}, 0 \leqslant$ $s \leqslant k$, которое является слоем интегрируемого нуль-распределения. Так как $\mu_{0}>k$, то каждый слой содержит стандартное комплексное проективное пространство $\mathbb{C P}^{1}$. Пусть $\gamma=\gamma(t),-\infty<t<+\infty,-$ геодезическая $\mathbb{C P}^{1}$ такая, что $\gamma(0)=Q_{0}$.

Конулевой оператор $C_{X}$ вдоль геодезической $\gamma$ удовлетворяет уравнению

$$
\frac{d C(t)}{d t}=C^{2}(t)+I_{2\left(l-\mu_{0}\right)}
$$

где $I_{2\left(n-\mu_{0}\right)}$ - тождественная матрица. Из результатов [33] следует, что уравнение (3.6.1) не имеет глобальных регулярных решений. Мы приходим к противоречию.

3.7. Сильно параболические подмногообразия и минимальные поверхности. Сильно параболические подмногообразия в евклидовом пространстве, которые одновременно являются минимальными, изучались в [18]. Трехмерное сильно 1-параболическое подмногообразие $F^{3}$ в $E^{n+1}$ имеет линейчатое строение: через каждую точку проходит прямолинейная образующая, вдоль которой касательная плоскость стационарна. Тогда мы имеем отображение $\Gamma: F^{3} \rightarrow S^{n}$, когда точка $Q \in F^{3}$, отображается в точку сферы, которая является концевой для единичного вектора, задающей направление прямолинейной образующей.

Пусть $V^{2}=\Gamma\left(F^{3}\right) \subset S^{n}$ будет регулярной поверхностью. Тогда геометрии $V^{2}$ и $F^{3}$ тесно связаны между собой. Имеет место

ТеОРема 3.7 .1 [18]. Пусть $F^{3}$ - сильно 1-параболическое подмногообразие, существенно лежсащее в $E^{n+1}(n>3)$, и образ $V^{2}=\Gamma\left(F^{3}\right)$ регулярен в $S^{n} \cdot F^{3}$ минимально в $E^{n+1}$ тогда и только тогда, когда $V^{2}$ минимально в $S^{n}$.

Верно и обратное: пусть $V^{2}$ - минимальная поверхность в $S^{n}$ и $\lambda$ - функция на $V^{2}$, удовлетворяющая уравнению

$$
\Delta \lambda+\lambda=0,
$$


где $\Delta$ - оператор Лапласа-Бельтрами на $V^{2}$. Тогда существует единственное, с точностью до параллельного переноса, минимальное сильно 1-параболическоеподмногообразие $F^{3}$ в $E^{n+1}$ такое, что

1) $V^{2}=\Gamma\left(F^{3}\right)$,

2) $\lambda$ - скручивающая функция для $F^{3}$.

Если $V^{2}$ есть полная минимальная поверхность в $S^{n}$, то $F^{3}$ является трехмерной плоскостью.

Эти результаты связаны с гармоническими морфизмами, ассоциированньми с минимальньми сильно $(k-2)$-параболическими $l$-мерными поверхностями в евклидовом пространстве. Имеется следуюший признак гармонических морфизмов: гладкое отображение

$$
f: M \rightarrow N
$$

является гармоническим морфизмом, если и только если оно одновременно гармонично и горизонтально конформно.

Имеет место

ТЕОРема 3.7.2 [19]. Пусть $F^{3}$ - сильно 1-параболическая поверхность в $E^{n+1}$ $u V^{2}=\Gamma\left(F^{3}\right) \subset S^{n}$. Тогда отображение $\Gamma: F^{3} \rightarrow V^{2}$ есть гармонический морфизм, если и только если выполняется одно из условий:

1) $F^{3}-$ конус над $V^{2}$,

2) $F^{3}-$ минимальна в $E^{n+1}$.

Верно обобщение этого утверждения на сильно $(l-2)$-параболические поверхности $F^{l} \subset E^{l+1}$.

Известна теорема Каталана о том, что полная линейчатая поверхность, отличная от плоскости в трехмерном евклидовом пространстве, есть геликоид. При обобщении этой теоремы на многомерньй случай появляются цилиндрические поверхности. Справедлива

ТЕОРема 3.7 .3 [39]. Пусть $F^{l}$ есть полная минимальная линейчатая гиперповерхность $c(l-1)$-мерными образующими в евклидовом пространстве $E^{l+1}$. Тогда или $F^{l}$ - гиперплоскость, или $F^{l}=F^{2} \times E^{l-2}$, где $F^{2}$ есть геликоид, т.е.

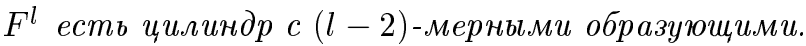

Обобщение теоремы Каталана на локальньй случай, т.е. на случай неполных поверхностей, дано в препринте [38].

Сильно параболические подмногообразия и их частный случай - вполне геодезические подмногообразия - появляются в классе полных минимальных подмногообразий.

С.Н. Берштейн доказал, что явно заданная в $\mathbb{E}^{3}$ уравнением $z=f(x, y)$ над всей плоскостью минимальная поверхность есть плоскость.

Для гиперповерхностей в $\mathbb{E}^{n}$ при $n \geqslant 9$ эта теорема не имеет места. Однако ее аналог справедлив для однозначно проектируемых минимальных гиперповерхностей в сферическом пространстве $S^{n}$.

Поверхность $F^{l} \subset S^{n}$ назьвается однозначно проектируемой, явно заданной, если сушествует вполне геодезическая большая сфера $S^{l} \subset S^{n}$ такая, что большие вполне 
геодезические сферы $S^{n-l}(Q)$, которые проходят через точки $Q \in F^{l}$ перпендикулярно $S^{l}$ и для которых пересечение $S^{n-l}(Q) \cap S^{l}$ состоит из одной точки, пересекаются с $F^{l}$ трансверсально [10].

Если из центра сферы $S^{n} \subset \mathbb{E}^{n+1}$ провести лучи через точки поверхности, то получим конус в $\mathbb{E}^{n+1}$, которьй имеет явное задание:

$$
\begin{gathered}
z_{1}=f_{1}\left(x_{1}, \ldots, x_{l+1}\right), \\
\ldots \ldots \ldots \ldots \ldots \ldots \ldots \\
z_{n-l}=f_{n-l}\left(x_{1}, \ldots, x_{l+1}\right) .
\end{gathered}
$$

А. В. Погорелов и Де Джорджи доказали, что явно заданные над всей сферой минимальные гиперповерхности в сферическом пространстве являются вполне геодезическими большими сферами [25], [22].

Двумерная явно заданная над сферой минимальная поверхность произвольной коразмерности в сферическом пространстве $S^{n}$ есть вполне геодезическая большая сфеpa [12].

Для минимальных поверхностей произвольной размерности и коразмерности в сферическом пространстве этот результат не имеет места [54].

\section{СПИСОК ЛИТЕРАТУРЫ}

[1] Акивис М. А., Рыжков В. В. Многомерные поверхности специальных проективных типов // Труды IV Всесоюзного математического съезда. Т. 2. Ленинград, 1964. С. 159-164.

[2] Аминов Ю.А. О грассмановом образе двумерной поверхности в четырехмерном евклидовом пространстве // Укр. геом. сб. Харьков. 1980. № 23. С. 3-16.

[3] Аминов Ю.А. Восстановление двумерной поверхности в $n$-мерном евклидовом пространстве по ее грассманову образу // Матем. заметки. 1984. Т. 35. № 2. С. 223-228.

[4] Арнольд В.И., Варченко А. Н., Гусейн-Заде С. М. Особенности дифференцируемых отображений. Т. 1. М.: Наука, 1982.

[5] Архангельский А. В. Конечномерные векторные пространства. М.: Изд-во МГУ, 1982.

[6] Борисенко А. А. О строении гиперповерхностей с нулевой мерой Хаусдорфа сферического изображения // Укр. геом. сб. Харьков. 1972. № 12. С. 36-45.

[7] Борисенко А. А. О строении $l$-мерных поверхностей с вырожденной второй квадратичной формой в $n$-мерном евклидовом пространстве // Укр. геом. сб. Харьков. 1973. № 13. C. $18-27$.

[8] Борисенко А.А. О строении непрерьвной поверхности, содержащей прямую // Укр. геом. сб. Харьков. 1973. № 14. С. 21-24.

[9] Борисенко А. А. О полных поверхностях в пространствах постоянной кривизны // Укр. геом. сб. Харьков. 1974. № 15. С. 8-15.

[10] Борисенко А. А. О внешне геометрических свойствах параболических поверхностей и топологических свойствах седловых поверхностей в симметрических пространствах ранга один // Матем. сб. 1981. Т. 116 (158). № 3 (11). С. 440-457.

[11] Борисенко А. А. О поверхностях неположительной внешней кривизны в пространствах постоянной кривизны // Матем. сб. 1981. Т. 114 (156). № 3. С. 339-354.

[12] Борисенко А.А. О явно заданных минимальных поверхностях // Укр. геом. сб. Харьков. 1982. №25. С. $6-7$.

[13] Борисенко А. А.О полных параболических поверхностях в евклидовом пространстве // Укр. геом. сб. Харьков. 1985. № 28. С. 8-19.

[14] Борисенко А.А. Аффинная классификация точек многомерных поверхностей // Сиб. матем. журн. 1990. Т. 31. № 3. С. 19-29. 
[15] Борисенко А. А. О цилиндрических многомерных поверхностях в пространстве Лобачевского // Укр. геом. сб. Харьков. 1990. №33. С. 18-27.

[16] Борисенко А. А., Николаевский Ю. А. Классификация точек трехмерных поверхностей по грассманову образу // Укр. геом. сб. Харьков. 1989. № 32. С. 11-27.

[17] Борисенко А. А., Остроумов С. А. О цилиндричности полных сильно параболических келеровых подмногообразий в комплексном эрмитовом пространстве // Мат. физика, анализ, геометрия. Харьков. 1995. Т. 2. № 3-4. С. 284-295.

[18] Борисенко А.А. (мл.) Силно параболические ассоциативные поверхности // УМН. 1993. T. 48. № 5. C. $165-166$.

[19] Борисенко А. А. (мл.) Гармонические морфизмы, ассоциированные с минимальными параболическими поверхностями в евклидовом пространстве // Вестник МГУ. 1996. №1. C. $3-12$.

[20] Глазырин В.В. Топологическое и метрическое строение $k$-седловых поверхностей // Сиб. матем. журн. 1978. Т. 19. № 3. С. 555-563.

[21] Громолл Д., Клингерберг В., Мейер В. Риманова геометрия в целом. М.: Мир, 1981.

[22] Де Джорджи Обобщение теоремы Бернштейна // Целочисленные потоки и минималшные поверхности. М.: Мир, 1973.

[23] Окрут С.И. Римановы гильбертовы подмногообразия неположительной внешней кривизны // Укр. геом. сб. Харьков. 1990. № 33. С. 77-91.

[24] Погорелов А. В. Поверхности ограниченной внешней кривизны. Харьков: Изд-во Харьк. ун-та, 1956.

[25] Погорелов А.В. О минимальных гиперповерхностях в сферическом пространстве // Докл. АН СССР. 1972. Т. 206. № 2. С. 291-292.

[26] Рыжков В.В. О тангенциально вырожденных поверхностях // Докл. АН СССР. 1960. T. 135. № 1. C. $23-25$.

[27] Топоногов В. А. Метрическое строение римановых пространств неотрицательной кривизны, содержащих прямую // Сиб. матем. журн. 1964. Т. 6. С. 1358-1370.

[28] Шефель С.З. Поверхности в евклидовом пространстве // Математический анализ и смежные вопросы математики. Новосибирск: Наука, 1978. С. 297-318.

[29] Шефель С.З. Геометрические свойства погруженных многообразий // Сиб. матем. журн. 1985. Т. 26. №1. С. 171-188.

[30] Шефель Г. С. Группы преобразований евклидова пространства // Сиб. матем. журн. 1985. T. 26. № 3. C. 197-215.

[31] Эйзенхарт Л.П. Риманова геометрия. М.: ИЛ, 1948.

[32] Abe K. A complex analogue of Hartman-Nirenberg cylinder theorem // J. Differential Geom. 1972,. V. 7. P. 453-460.

[33] Abe K. Application of a Riccati type differential equation to Riemannian manifolds with totally geodesic distributions // Tôhoku Math. J. 1973. V. 25. P. 425-444.

[34] Abe K. Some remarks on a class of submanifolds in space forms of nonnegative curvature // Math. Ann. 1980. V. 247. №3. P. 275-278.

[35] Abe K., Magid M. A. Relative nullity foliations and indefinite isometric immersions // Pacific J. Math. 1986. V. 124. № 1. P. 1-20.

[36] Abe K., Magid M. A. Indefinite Kahler submanifolds with positive index of relative nullity // Ann. Global Anal. Geom. 1988. V. 6. № 3. P. 231-258.

[37] Borisenko A. A., Rabelo M., Tenenblat K. Strongly parabolic timelike submanifolds of Minkovsky space // Preprint, 1996.

[38] Barbosa S. L., do Carno M. Helicoids, catenoids and minimal hyperfaces of $R^{n}$ invariant by an $l$-parameter group of motions // An Acad. Brasil. Cbenc. Preprint, 1983.

[39] Blair D. E., Vanstone S. R. A generalization of the helicoid. Minimal submanifolds and geodesies // Proc. Japan-USA Sem. Tokyo. 1977. Amsterdam: Noth-Holland, 1978. P. $13-16$.

[40] Cartan E. Sur les varietés de courbure constante d'un espace euclidien ou noneuclidien // Bull. Soc. Math. France. 1919. V. 47; 1920. V. 48. 
[41] Hartman P. On the isometric immersion in Euclidean space of manifolds with nonnegative sectional curvature // Trans. Amer. Math. Soc. 1970. V. 147. № 2. P. 541-561.

[42] Hartman P., Nirenberg L. On spherical image maps whose jacobians do not change sign // Amer. J. Math. 1959. V. 81. P. 901-920.

[43] Cheeger J., Gromoll D. The splitting theorem for manifolds of nonnegative Ricci curvature // J. Differential Geom. 1971. V. 6. № 1. P. 119-129.

[44] Chen B.Y. Geometry of submanifolds and its applications. V. III. Tokyo, 1981.

[45] Chern S.S., Kuiper N. H. Some theorems on the isometric imbedding of compact Riemann manifold in Euclidean space // Ann. Math. 1956. V. 56. P. 422-430.

[46] Dajczer M., Gromoll D. On spherical submanifolds with nullity // Proc. Amer. Math. Soc. 1985. V. 93. № 1. P. 99-100.

[47] Dajczer M., Gromoll D. Gauss parametrizations and rigity aspects of submanifolds // J. Differential Geom. 1985. V. 22. №1. P. 1-13.

[48] Dajczer M., Gromoll D. Rigity of complete Euclidean hypersurfaces // J. Differential Geom. 1990. V. 31. P. 401-416.

[49] Ferus D. On the type number of hypersurfaces // Math. Ann. 1970. V. 187. P. 310-316.

[50] Ferus D. Totally geodesic foliations // Math. Ann. 1970. V. 188. P. 313-316.

[51] Ferus D. On isometric immersions between hyperbolic spaces // Math. Ann. 1973. V. 205. P. 193-200.

[52] Florit L. A splitting theorem for Euclidean submanifolds of nonpositive sectional curvature // Preprint, 1996.

[53] Graves L. Codimension one isometric immersions between Lorentz spaces // Trans. Amer. Math. Soc. 1979. V. 252. P. 367-392.

[54] Lawson H. B., Osserman R. Non existence, non-uniqueness and irregularity of solutions to the minimal surface system // Acta Mat. 1977. V. 139. № 1. P. 1-17.

[55] Maltz R. The nullity spaces of curvature like tensors // J. Differential Geom. 1972. V. 7. № 3-4. P. 519-523.

[56] Newman A.C. A proof of the splitting conjucture of S. T. Yau // J. Differential Geom. 1990. V. 31. № 1. P. 163-184.

[57] Nomizu K. Isometric immersions of the hyperbolic plane into the hyperbolic space // Math. Ann. 1973. V. 205. P. 181-192.

[58] Rosental A. Riemannian manifolds of constant nullity // Michigan Math. J. 1967. V. 14. P. 469-480.

[59] Rosental A. Kahler manifolds of constant nullity // Michigan Math. J. 1968. V. 15. P. 433-440.

[60] Sacksteder R. On hypersurfaces with no negative sectional curvatures // Amer. J. Math. 1960. V. 82. № 3. P. 609-630. 